\title{
A Bayesian approach for wavenumber identification of metamaterial beams possessing variability
}

\author{
Marcos. R. Souza ${ }^{a}$, Danilo Beli ${ }^{\text {bcc }}$, Neil S. Ferguson ${ }^{\mathrm{a}}$, José Roberto de F. Arruda ${ }^{\mathrm{b}}$, Adriano T. Fabro ${ }^{\mathrm{d}, *}$ \\ ${ }^{a}$ ISVR, University of Southampton, Southampton, UK \\ ${ }^{\mathrm{b}} \mathrm{School}$ of Mechanical Engineering, University of Campinas, Campinas, Brazil \\ ${ }^{\mathrm{c} S a ̃ o}$ Carlos School of Engineering, University of São Paulo, São Carlos, Brazil \\ ${ }^{\mathrm{d}}$ Department of Mechanical Engineering, University of Brasilia, Brasilia, Brazil
}

\begin{abstract}
Recent developments in additive manufacturing have allowed for a number of innovative designs in elastic metamaterials and phononic crystals used in several applications, including vibration attenuation. Complex geometric patterns that were otherwise very expensive or unpractical to produce are currently feasible. However, the 3D printing also introduces variability, which can greatly affect the dynamic performance of the metastructure. This work investigates the effects of manufacturing variability on the wavenumber identification of beams with evenly attached resonators, produced from Selective Laser Sintering. A combination of a correlation-based technique and a Bayes framework is proposed to identify the effective wavenumber and the most probable values of some of the design parameters. Typically of interest, for vibration attenuation using metamaterials, are the mass ratio and the resonator natural frequency. For this purpose an analytical model is derived, assuming an infinite number of resonators tuned to the same frequency. These parameters can be highly affected by the manufacturing variability because they are dependent on complex geometrical features of the metastructure. It is shown that the proposed approach can estimate the most likely values of the parameters with less than $4 \%$ difference when compared to a benchmark approach; the latter is not only more complex and time demanding, but also based on indirect measurements. Understanding the effects of this variability on the wave propagation represents an important step towards proposing robust designs with respect to the attenuation performance.
\end{abstract}

Keywords: Bayesian estimation, wavenumber identification, uncertainty quantification, band gap, elastic metamaterial

\section{Introduction}

Recently, metamaterials and phononic crystals, which can be described as a class of structures designed to manipulate acoustic and elastic waves [1], have been applied for vibration attenuation purposes (e.g. [2,3]). Most of the recent developments in elastic metamaterials are due to the currently available 3D printers [4-6]. However, like any other manufacturing process, the 3D printers introduce manufacturing variability with respect to the nominal periodic design $[7,8]$ which has been shown to greatly affect the metamaterial vibration attenuation performance $[9,10]$, leading to wave trapping and band gap widening [11-13] or annihilation [14]. Very limited experimental work is available in the literature concerning the investigation of the dynamic performance of 3D printed structures and the estimation of their mechanical or geometric parameters (e.g. [15]). In this sense, there is a growing need for more experimental investigation into this subject. 
The Bayesian approach, based on Bayes' theorem, has been used as tool to infer system parameters from a given set of data[16] in the context of inverse problems. Regardless of the fact that parameters of a model may have a deterministic value, a Bayesian approach allows for the treatment of parameters as uncertain, providing a method that reflects one's uncertainty about the true value of the parameters. It can be interpreted as a 'statement of belief' based on expert opinion [17]. A Bayesian approach can also take into account the findings and knowledge of previous work in the form of the prior distribution, whilst a more classical statistical approach usually cannot go further than using previous research to assist the formulation of hypotheses[18]. This is in contrast to the frequentist approach interpretation of probability, which assumes an inherited randomness of certain random variables associated with a random phenomenon [19].

In computational mechanics the Bayesian approach has been used in several applications, such as for the identification of geometrical or mechanical properties [20,21], damage detection or assessment [22,23], or as a tool for model selection [24,25], i.e., choosing a mechanical model that best represents a set of measurements. Wave-based approaches have also been used for the identification of mechanical properties using ultrasound [26,27], which requires measuring the wave speed, or by directly estimating the dispersion relation [28-31] using frequency response function measurements. Bayesian approaches have previously been proposed for characterizing elastic constants in composite materials using ultrasonic Lamb waves [32], and in electrical cable bundles [33] using dispersion relationships corresponding to flexural waves. However, to the best of the authors' knowledge, it has not yet been applied to the identification of properties of locally resonant metamaterials.

This work aims at investigating the effects of manufacturing variability on the effective wavenumber identification in beams with evenly attached resonators produced from Selective Laser Sintering (SLS). A combination of a correlation technique[34,35] applied to frequency response measurements and a Bayesian framework is proposed to identify the wavenumber and most probable values of some of the design parameters typically of interest for vibration attenuation using metamaterials, namely the mass ratio and the natural frequencies of the resonators. For this purpose, an analytical model is derived assuming an infinite number of resonators tuned to the same frequency based on the work by Sugino et al.[14,36]. These parameters can be affected by the manufacturing variability due to the complex geometrical features of the metastructure. The dispersion relation of an equivalent EulerBernoulli beam model is used at lower frequencies to identify the Young's modulus of the metamaterial host beam. It is shown that the proposed approach can estimate the most probable values of the parameters with less than less than $4 \%$ difference when compared to a benchmark approach proposed by Beli et al.[10]. The latter is more complex and time demanding and is based on indirect measurements, but provides the complete set of properties along the space. Understanding the effects of this variability on the wave propagation represents an important step towards proposing robust designs with respect to the attenuation performance.

This paper is organized as follows. Section 2 presents a bending wavenumber expression for the metastructure, which is then used along with a Bayesian parameter identification strategy presented in Section 3, which also describes the proposed approach using synthetic data, produced from numerical simulations. Section 4 presents the results of the proposed approach from experimental measurements. Finally, Section 5 gives some concluding remarks.

\section{Wave motion: dispersion analysis}

In this section, an equivalent bending wavenumber expression is presented for the case of a continuous host structure with periodically attached undamped lattice resonators. This result is used throughout the paper in the identification approach. 
It has been shown that the actual working mechanism for vibration attenuation in metamaterials is related to the vibration absorber principle [37], known as Mie-type or Fabry-Perot-type resonances [38], while the working mechanism of phononic crystals is related to the periodicity in the material and geometrical properties [39], known as the Bragg scattering effect. For practical engineering applications, only a limited number of resonators along the metastructure is feasible. In this sense, Sugino et al. [14,36] discussed the optimum number of resonators and the importance of the resonator positioning, considering the targeted frequency band, the mass ratio and the boundary conditions, in terms of a Riemann sum. They showed that the optimum band gap is equivalent to the assumption of an infinite number of attached resonators.

An analytical expression can be derived based on a continuous beam model with point-attached lumped parameter resonators. Assuming that the equations of motion of a continuous Euler-Bernoulli (EB) beam undergoing flexural vibration with $S$ periodically point attached undamped resonators, with translation and no rotational inertia, can be given in general form by $[14,36]$

$$
E I \frac{\partial^{4} w(x, t)}{\partial x^{4}}+\mu \frac{\partial^{2} w(x, t)}{\partial t^{2}}-\sum_{p=1}^{S} k_{p} u_{p}(t) \delta\left(x-x_{p}\right)=p(x, t),
$$

and, for the resonators,

$$
m_{p} \frac{\partial^{2} u_{p}(t)}{\partial t^{2}}+k_{p} u_{p}(t)+m_{p} \frac{\partial^{2} w\left(x_{p}, t\right)}{\partial t^{2}}=0,
$$

where $w(x, t)$ is the transverse displacement of the beam due to bending at position $x$ and time $t, E I$ is the bending stiffness, $\mu$ is the host beam mass per unit length, $k_{p}$ and $m_{p}$ are the lumped stiffness and mass of the $p^{t h}$ resonator attached at $x_{p}$ position, $p(x, t)$ is the distributed load and $\delta(x)$ is the Dirac delta function. This expression was originally proposed for a modal analysis of metastructures aimed at the derivation of a closed form expression for the frequency edges of the band gap, and has been shown to be equivalent to a metastructure with the optimum number of resonators $[14,36]$. In this work, it is used for finding a simplified equivalent dispersion equation, i.e., the metastructure is modelled as a continuous structure with an equivalent wavenumber rather than a periodic structure with a given phase change.

Assuming time harmonic motion, i.e. $w(x, t)=W(x) e^{i \omega t}, p(x, t)=P(x) e^{i \omega t}, u_{p}(t)=U_{p} e^{i \omega t}$ for the $p^{\text {th }}$ resonator, the displacement of the $p^{\text {th }}$ resonator can be related to the beam displacement at $x_{p}$ by

$$
U_{p}=\frac{\omega^{2}}{\omega_{p}^{2}-\omega^{2}} W\left(x_{p}\right)
$$

where $\omega_{p}$ is the natural frequency of the $p^{\text {th }}$ resonator. Assuming identical resonators, it is possible to show that

$$
E I \frac{d^{4} W(x)}{d x^{4}}-\omega^{2} \mu W(x)-\epsilon \mu \omega^{2} \frac{\omega_{r}^{2}}{\omega_{r}^{2}-\omega^{2}} \sum_{p=1}^{S} W\left(x_{p}\right) \delta\left(x-x_{p}\right) \Delta l=P(x),
$$

where $\epsilon$ is the mass ratio, defined as the ratio of the mass of the resonators by the mass of the host beam, and $\omega_{r}$ is the natural frequency of the identical resonators and $\Delta l$ is the spacing between resonators. Assuming that $S \rightarrow \infty$, such that the summation becomes an integral in the Riemann sense, and writing the displacement field in the terms of space harmonics, i.e. $W(x)=\widehat{W} e^{-i k x}$, thus 
where $\Omega_{r}=\omega / \omega_{r}$ is the ratio of the natural frequency of the resonators $\omega_{r}$ and the excitation frequency $\omega$. Details of this derivation are given in Appendix A.1. The wavenumber can be real, meaning a propagating wave, imaginary, meaning a decaying or evanescent wave, or complex, meaning a propagating wave with decay. The imaginary part of the dispersion curve (negative values) shows the frequency band in which there is vibration attenuation, i.e. the band gap for the flexural waves. This is caused by the resonators and it is known as locally resonant band gap[1].

\section{Parameter identification strategy}

Experimental data can be used to identify the dynamical properties of a structure. Classically, this is done by curve fitting experimental frequency response functions (FRFs) using a modal model to identify modal parameters, i.e., natural frequencies, modal damping, and mode shapes [40-44]. This would require a previous knowledge about the boundary conditions of the measured structure or, at least, they would need to be assumed. The spectral element method[15], although considered a wavebased approach, also requires the definition of boundary conditions. Dispersion curves have been used with a Bayesian approach in some engineering applications, for instance for estimating mechanical parameters in composites [32,45], water pipelines [46] and cable bundles [33]. Despite applying different wave measurement methodologies, they typically use the wavenumber estimation to fit mechanical parameters under some Bayesian framework. In this paper, a correlation technique[34,35] is used to estimate the dispersion curves of a beam with attached resonators from the measured FRFs. One advantage of identifying the dispersion curve from a correlation technique is that this method does not require any prior knowledge of the structure, such as boundary conditions, material properties or cross-sectional dimensions[47], while not being very sensitive to sensor placement [31].

This paper combines the use of a correlation technique to estimate dispersion curves for a beam with a set of nominally identical resonators with a Bayesian approach, in order to obtain the most likely dispersion curve for the beam. Then, from the inferred dispersion curve, one can identify the Young's modulus of the beam along with its mass ratio, i.e. the ratio between the mass of the beam and the mass of the resonators, and the natural frequency of the resonators.

\subsection{Bayesian identification}

Bayesian inference is used in order to update the prior probability density function (PDF) [48] of the bending wavenumber of a beam with a set of resonators. Assuming that the only knowledge available about the prior distribution is its lower and upper limits, a uniform probability distribution gives the maximal uncertainty for a random variable. In other words, if there are no other strong grounds for assuming a different prior, a uniform distribution is the best initial assumption [24]. That is a result of the Maximum Entropy Principle [49-51]. Any other information considered about the prior distribution results in a non-uniform distribution, meaning that the considered random variables have less entropy or, in another words, more information, which would put less weight on the role of the experimental data updating the prior distribution [25].

The measured data is usually assumed to be contaminated with additive Gaussian noise in the form of $\mathbf{y}(\boldsymbol{\theta})=\mathbf{q}(\boldsymbol{\theta})+\mathbf{e}$, where $\mathbf{q}(\boldsymbol{\theta})$ is the system output of a model under the same excitation $\boldsymbol{\theta}, \mathbf{y}(\boldsymbol{\theta})$ is the measured data and $\mathbf{e}$ is the prediction error, which accounts for the difference between $\mathbf{y}(\boldsymbol{\theta})$ and 
$\mathbf{q}(\boldsymbol{\theta})[52,53]$. In a Bayesian context, assuming the measured data $\mathbf{y}=\left[y_{1}, y_{2}, \ldots, y_{N}\right]$, the posterior distribution of the set of model parameters $\boldsymbol{\theta}$, given the data $\mathbf{y}$, is[52]

$$
p(\boldsymbol{\theta} \mid \mathbf{y})=\frac{p(\mathbf{y} \mid \boldsymbol{\theta}) p(\boldsymbol{\theta})}{p(\mathbf{y})},
$$

where $p(\mathbf{y} \mid \boldsymbol{\theta})$ is the likelihood function, $p(\boldsymbol{\theta})$ is the prior distribution and $p(\mathbf{y})$ is a normalising constant. In other words, at every frequency, $p(\mathbf{y} \mid \boldsymbol{\theta})$ is the probability of the observed data $k_{B_{\text {observed }}}$, given a wavenumber $k_{B}$ from the prior distribution, which is assumed to be uniform. The prior, $p(\boldsymbol{\theta})$, is the distribution of the modelled wavenumber $k_{B}$. The function $p(\mathbf{y})$ is the PDF of the observed data and can be treated as a simple normalising constant that does not affect the identification of the parameter of interest.

\subsection{Synthetic data}

In this section, a numerical simulation is proposed as a proxy for experimental data in order to assess the applicability of the Bayesian inference. Twenty dispersion curves were produced with the introduction of an additive noise to the actual exact wavenumbers $k_{B}(\omega)$ in the form of $k_{B_{\text {synth }}}=$ $k_{B}[1+e(\omega)]$. In this pseudo-experiment, $k_{B_{\text {synth }}}$ represents $k_{B_{\text {observed }}}$. Figure 1shows one sample of the synthetic dispersion curve and the nominal wavenumber, from Eq. (6). The general properties used for the synthetic data are given in Table 1, which corresponds to the nominal values of the metastructure presented in Appendix A.2.

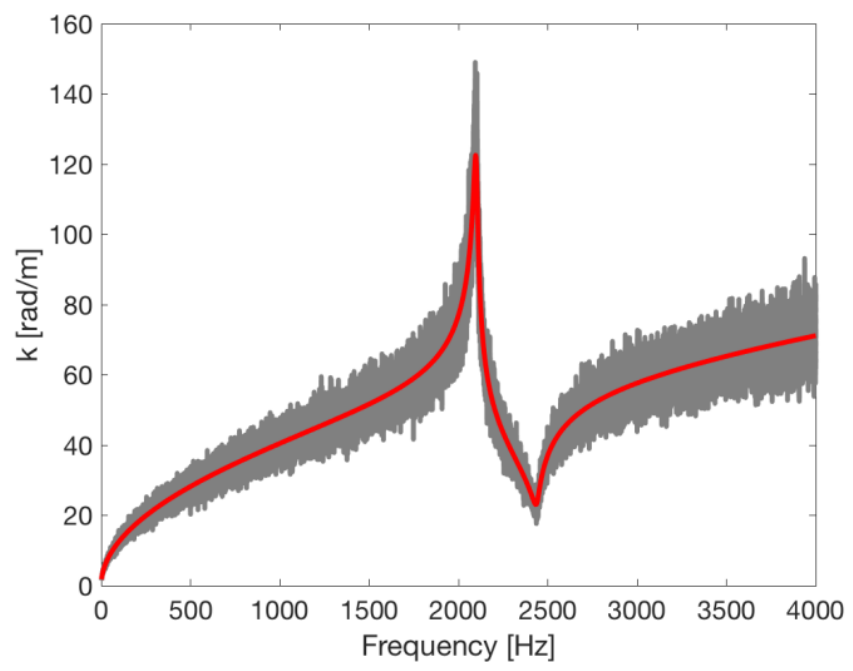

Figure 1- Synthetic data and synthetic data polluted with noise. Nominal case (-) and the twenty synthetic cases (-).

Table 1 - Mechanical and geometrical properties of the metamaterial beam used for the synthetic data.

\begin{tabular}{c|cccccccc} 
Property & $\begin{array}{c}E \\
{[\mathrm{GPa}]}\end{array}$ & $\begin{array}{c}I \\
{\left[\mathrm{~m}^{4}\right]}\end{array}$ & $\eta$ & $\begin{array}{c}\rho \\
{\left[\mathrm{kg} / \mathrm{m}^{3}\right]}\end{array}$ & $\begin{array}{c}A \\
{\left[\mathrm{~m}^{2}\right]}\end{array}$ & $\begin{array}{c}f_{r} \\
{[\mathrm{~Hz}]}\end{array}$ & $\begin{array}{c}L \\
{[\mathrm{~m}]}\end{array}$ & $\epsilon$ \\
\hline Value & 1 & $2.048 \times 10^{-9}$ & 0.01 & 1000 & $9.6 \times 10^{-5}$ & 2100 & 0.33 & 0.35
\end{tabular}


The likelihood function is often formulated in terms of the negative log-likelihood function[52]. Assuming $\boldsymbol{e}$ to be independent and Gaussian distributed with zero mean and standard deviation $\sigma_{S}$, it is possible to write[52]

$$
p(\mathbf{y} \mid \boldsymbol{\theta})=\prod_{i=1}^{N} \frac{1}{\sqrt{2 \pi} \sigma_{S}} e^{\left\{\frac{-1}{2 \sigma_{S}^{2}\left[\mathbf{y}_{i}(\boldsymbol{\theta})-\mathbf{q}_{i}(\boldsymbol{\theta})\right]^{2}}\right\} .}
$$

In this particular case, $\boldsymbol{y}_{i}(\boldsymbol{\theta})$ are the synthetic bending wavenumbers $k_{B_{s y n t h}}$ at a given frequency $\omega$ and $\mathbf{q}_{\boldsymbol{i}}(\boldsymbol{\theta})$ is the bending wavenumber $k_{B}$ sampled from the uniform prior distribution. The uniform prior distribution assumes that $k_{B}$ is between 0 and $200 \mathrm{rad} / \mathrm{m}$. The analysis is made with a frequency spacing of $2 \mathrm{~Hz}$, sampling a wavenumber value from the prior and using it as $\mathbf{q}(\boldsymbol{\theta})$.

For a given index $i$, there is an associated value for $k_{B}$ that comes from the assumed uniform prior distribution, while $\mathbf{y}_{i}(\boldsymbol{\theta})$ is given from the synthetic data. Therefore, in this example, for every index $i, \mathbf{y}_{i}(\boldsymbol{\theta})$ is a vector with $N=20$ entries for the wavenumber from the pseudo experiment. This is enough to achieve convergence of the estimated parameters. Every individual entry of vector $\mathbf{q}_{\boldsymbol{i}}(\boldsymbol{\theta})$ is identical to $k_{B}$, such that it has $N$ elements and matches the size of vector $\mathbf{y}_{i}(\boldsymbol{\theta})$. Finally, it is possible to find a likelihood function $p(\boldsymbol{y} \mid \boldsymbol{\theta})$ associated with each index $i$. At every $i$, equivalent to a $k_{B}$ value, the value of the prior distribution is multiplied by the value of the likelihood distribution, resulting in a value proportional to the posterior distribution, Eq. (6). For purposes of parameter identification, it is only necessary to know which $k_{B}$ (or index $i$ ) maximises the result of this multiplication, equivalent to the Bayes' factor [54]. This approach is also known as the maximum a posteriori estimator (MAP) [55]. Figure 2 shows the identified probability of the wavenumber for each frequency obtained via Bayesian inference from the synthetic data. The light yellow colour shows the wavenumbers with higher probability of representing the actual beam wavenumber while the dark blue colour indicates the corresponding wavenumbers with lower probability as a function of frequency. The inferred result is then used to estimate the Young's modulus $E$, the mass ratio $\epsilon$ and the natural frequency of the resonators $\omega_{r}$.

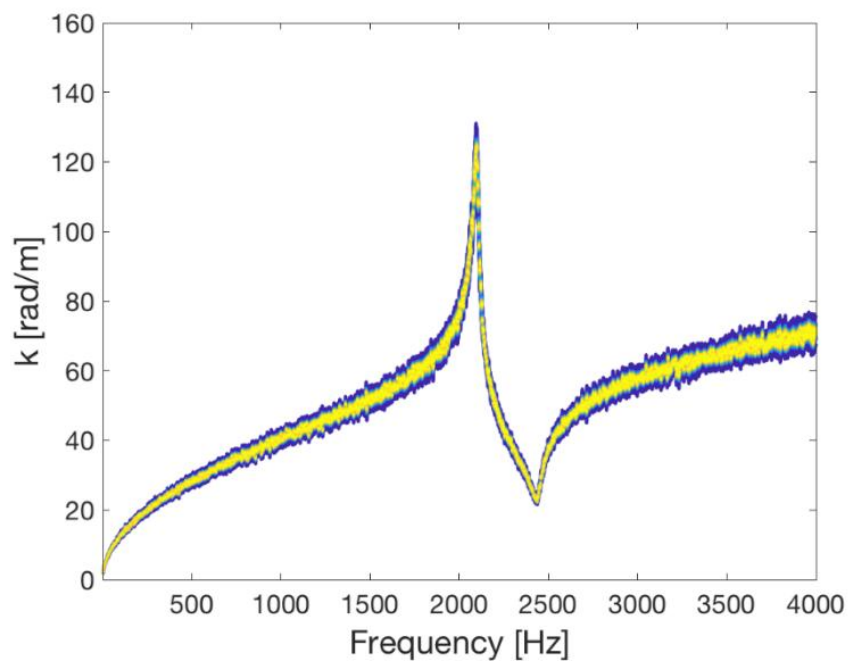

Figure 2- Dispersion curve identified via Bayesian inference from the synthetic data. Light colours indicate higher probability while dark blue indicates lower probability. 
For the estimation of the parameters, it is assumed that at frequencies lower than the band gap additional mass due to the resonators. Therefore, the density of the equivalent EB beam is given by the sum of the mass of the beam plus the mass of the resonators, i.e.

$$
\tilde{\rho}=\frac{m_{\text {beam }}+m_{\text {resonatos }}}{A L}=\rho(1+\epsilon),
$$

where $\tilde{\rho}$ is the density of the equivalent EB beam, $A$ is the cross-section area, $L$ is the length, $\rho$ is the density of the host or baseline beam and $\epsilon$ is the mass ratio. Figure 3(a) shows the dispersion curves of the beam with added resonators and the equivalent beam using nominal properties. Figure 3(b) presents the ratio of both dispersion curves. It can be noted that both dispersion curves are identical for frequencies lower than $\omega_{r} / 2$, half the natural frequency of the resonators.

(a)

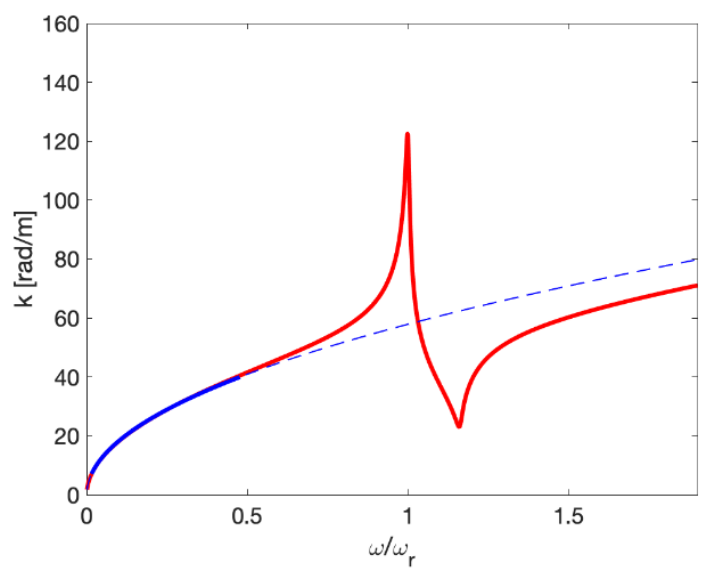

(b)

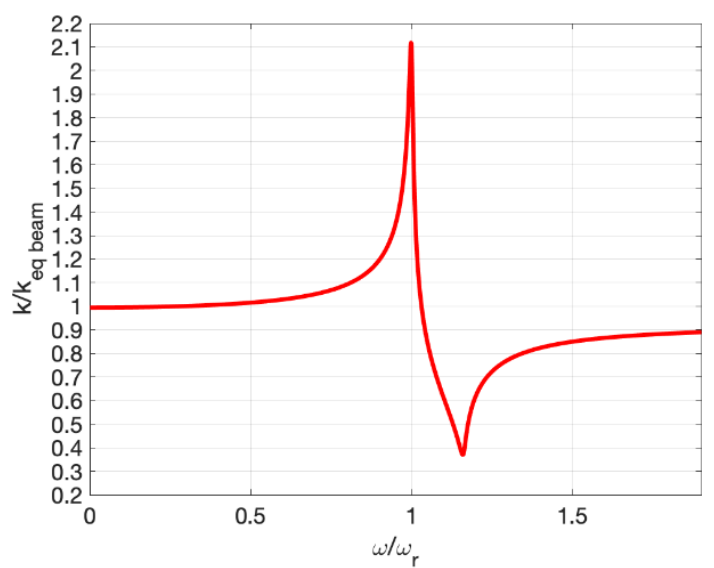

Figure 3- (a) Dispersion curves of the beam with resonators (red full line) and for the equivalent bare beam (dashed blue line). The frequency range used to identify the Young's modulus is highlighted (solid blue line). (b) Ratio of the wavenumber of the metamaterial to that of the equivalent beam.

The Young's modulus of the beam is then given by

$$
E=\frac{\tilde{\rho} A \omega^{2}}{I \tilde{k}^{4}}
$$

where $\tilde{k}$ is the wavenumber of the equivalent EB beam in the frequency range considered. Using the values inferred for the dispersion curve from the Bayesian approach and Eq. (9), in the range between $16 \mathrm{~Hz}$ and $1 \mathrm{kHz}$,it is possible to calculate the Young's modulus of the equivalent EB beam for every frequency, as shown in Figure 4 (a). It is assumed that the Young's modulus is not frequency dependent. Therefore, a probability distribution can be estimated for the Young's modulus. Figure 4 (b) presents the histogram obtained for the Young's modulus. It is expected that the Young's modulus distribution is not Gaussian due to the non-linear mapping from the random variable $\tilde{k}$ to the random variable $E$, Eq. (9). For the sake of simplicity, the Maximum Entropy argument [51,56] is applied to fit a Gamma distribution to the data. It is presented in Figure 4 (b). The Gamma distribution is of the type $p_{E}(x)=\left(1 / \vartheta^{\kappa} \Gamma(\kappa)\right) x^{(\kappa-1)} \exp (-x / \vartheta)$, where $\kappa$ is the shape factor and $\vartheta$ is the scale factor. The identified value for the Young's modulus from this procedure is given by the mean value of the fitted Gamma distribution.

The mass ratio $\epsilon$ and the natural frequency $f_{r}=\omega_{r} / 2 \pi$ of the resonators are obtained using a nonlinear least squares approach to fit the data to the model in Eq. (5) and using the identified Young's modulus. The curve fit was made in the frequency range between $1 \mathrm{kHz}$ and $4 \mathrm{kHz}$. These values 
were chosen after inspection of the dispersion curves. The lower limit was chosen in order to be roughly above $\omega_{r} / 2$, which is also the upper limit for the estimation of the Young's modulus. The upper limit was set to be above the band gap. A loss factor of $1 \%$ was considered in the dispersion curves. Results are summarized in Table 2, and present a maximum error of $2.1 \%$ for the Young's modulus.

(a)

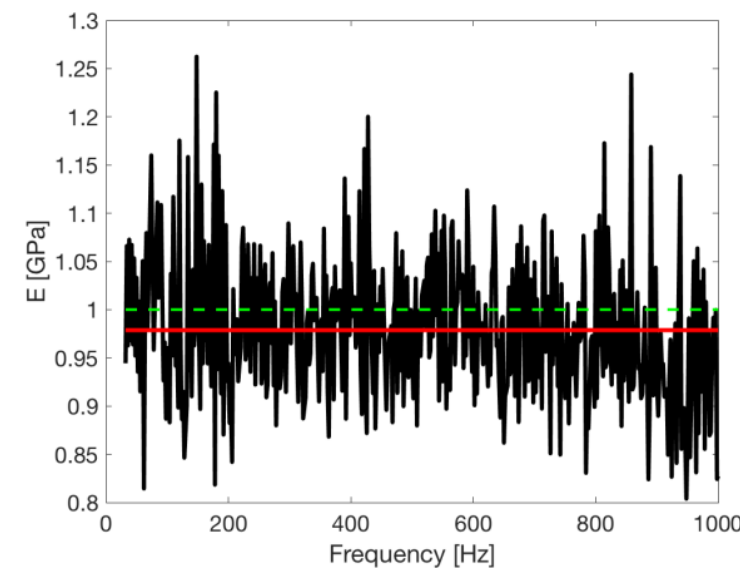

(b)

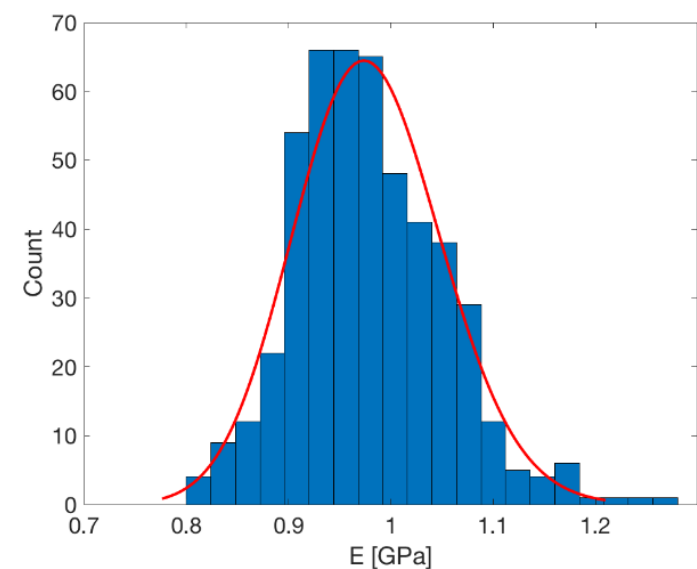

Figure 4- (a) Young's modulus from the Bayesian inferred dispersion curve and the equivalent EB beam. Calculated (black full line), actual (dashed green line) and identified (solid red line) Young's modulus. (b) Histogram of the Young's modulus and the fitted Gamma distribution with shape factor $\kappa=183.73$ scale factor $\vartheta=5.327 \times 10^{6}$.

Table 2 - Nominal and identified Young's modulus, resonator natural frequency and mass ratio from synthetic data.

\begin{tabular}{c|ccc} 
& $E$ & $f_{r}$ & \\
& {$[\mathrm{GPa}]$} & {$[\mathrm{Hz}]$} & $\epsilon$ \\
\hline Nominal & 1.00 & 2100 & 0.350 \\
Identified & 0.98 & 2080 & 0.349 \\
error & $2.1 \%$ & $0.95 \%$ & $0.28 \%$
\end{tabular}

Figure 5 presents the dispersion curve obtained with nominal values, with the identified parameters and the Bayesian inferred. A very good match is observed and shows that the proposed approach is suitable. In the next section, the proposed approach is applied to a set of experimental FRFs from a single metamaterial beam, which was produced using SLS 3D printing.

Note that the presented numerical experiment can have two distinct interpretations. The first considers that every $k_{B_{\text {synth }}}$ is estimated for a single beam from a set of nominally identical beams. Therefore, the Bayesian estimation gives the most probable parameters representing the ensemble of beams. A second point of view is that every $k_{B_{\text {synth }}}$ is generated from different positions along a single beam with non-uniform properties along its length, i.e. a near periodic beam. Therefore, the Bayesian estimation gives the most probable parameters representing a single periodic structure. The latter is used for applying the proposed approach to the experimental data presented in the next section. 


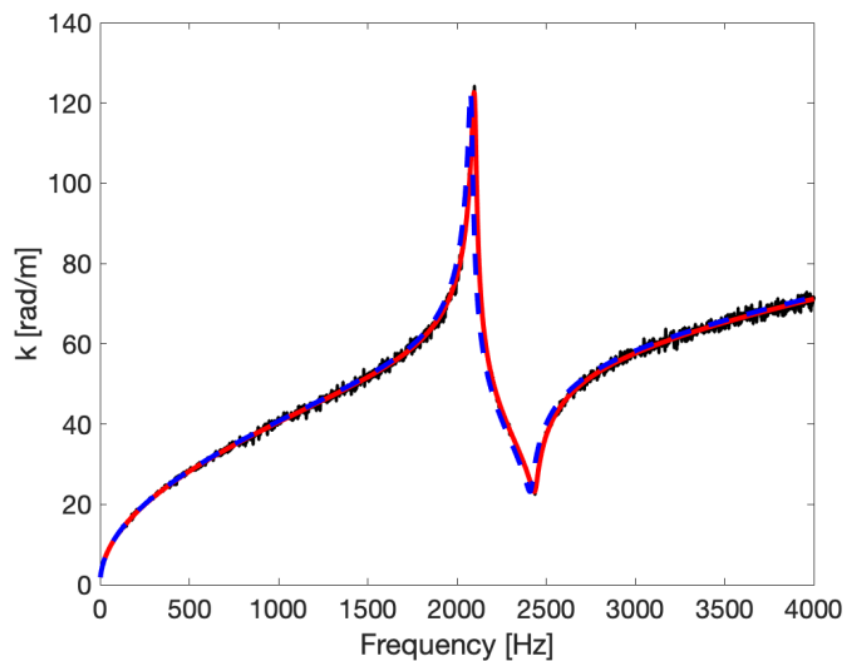

Figure 5-Dispersion curve obtained with nominal values (dashed blue line), with the identified parameters (solid red line) and the Bayesian inferred (black line).

\section{Experimental data}

In this section, the proposed Bayesian identification approach is applied to estimate the effective dispersion curve of the metamaterial beam presented in Appendix A.2 from the experimental FRFs obtained with the set-up presented in Appendix A.3. The wavenumbers are obtained from a correlation-based wavenumber identification technique [34,35]. A total of 59 FRFs were measured along the length of one beam. Each FRF can be considered as an independent experiment. Therefore, the correlation technique followed by the Bayesian inference can be applied on different subsets of these 59 FRFs. Assuming that $x=0$ is at the midpoint of the beam along the axis of propagation, the FRFs used were measured between the points $x=-0.11$ and $x=0.11$, resulting in a total of 41 FRFs available for the correlation technique.

From the 41 FRFs available, see Figure 6, 25 were randomly chosen from a uniform distribution to perform the correlation and estimate the effective wavenumber. This procedure was performed 20 times, thus creating the ensemble of measurement sets. Each of these twenty estimations generates a dispersion curve, which is then used as an input for the Bayesian inference.

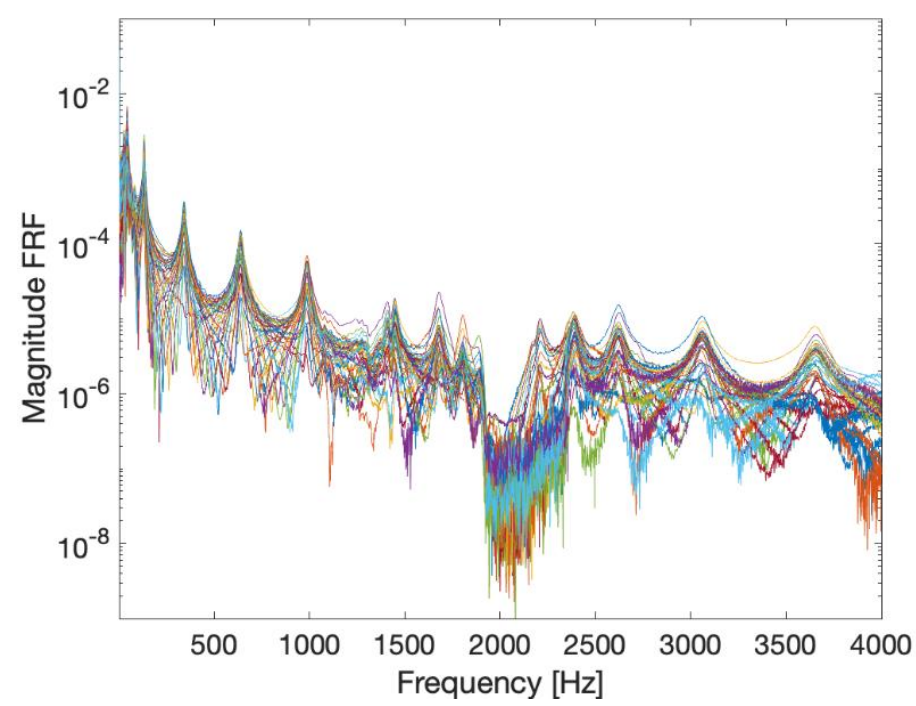

Figure 6-Experimentally obtained FRFs. 
The two-dimensional correlation-based wavenumber identification approach [34,35] can be

where $N_{p}$ is the number of points measured, $Y\left(x_{j}, \omega\right)$ is the complex transfer function measured at $x=x_{j}$ at frequency $\omega$ and $k_{t x}$ is the trial wavenumber to be estimated. Recall that the wavenumber can be real, meaning a propagating wave, imaginary, meaning a decaying or evanescent wave, or complex, meaning a propagating wave with decay. The trial wavenumbers are real numbers with arbitrarily fine resolution, unlike the typical Discrete Fourier Transform approach where the spatial frequency resolution is determined by the total length of the measurement.

Note that the measurement points are chosen around the midspan to avoid the effects of the ends of the beam in the identification technique. Typically, evanescent waves are important only close to boundaries and discontinuities and are not represented with the propagating wave type of field used in Eq. (10). The number of measurement point gives the spatial resolution and, therefore, the maximum and minimum wavenumber that can be identified. For the low wavenumber limit, the spatial measurement length has to cover at least half the wavelength, while the maximum wavenumber is defined by the spatial resolution[34]. Moreover, the measurement points are not required to be symmetric or evenly spaced along the beam. The choice of points is such that the wavenumber can be accurately estimated within the frequency band of interest. Moreover, the presence of large damping has the effect of greatly reducing the amplitude of the travelling waves and, therefore, impairs the effectiveness of the correlation technique. In this case, waves tend to die out rapidly from the excitation point location and little information about positive and negative going waves remain in the displacement field. An alternative, McDaniel's approach [47], has been shown to be effective for estimating wavenumbers in highly damped structures [57]. The correlation technique used in this work is typically suitable for lightly damped structures. A typical result of the correlation technique for an individual beam is shown in Figure 7. Negative and positive wavenumbers indicate different directions of the travelling waves.

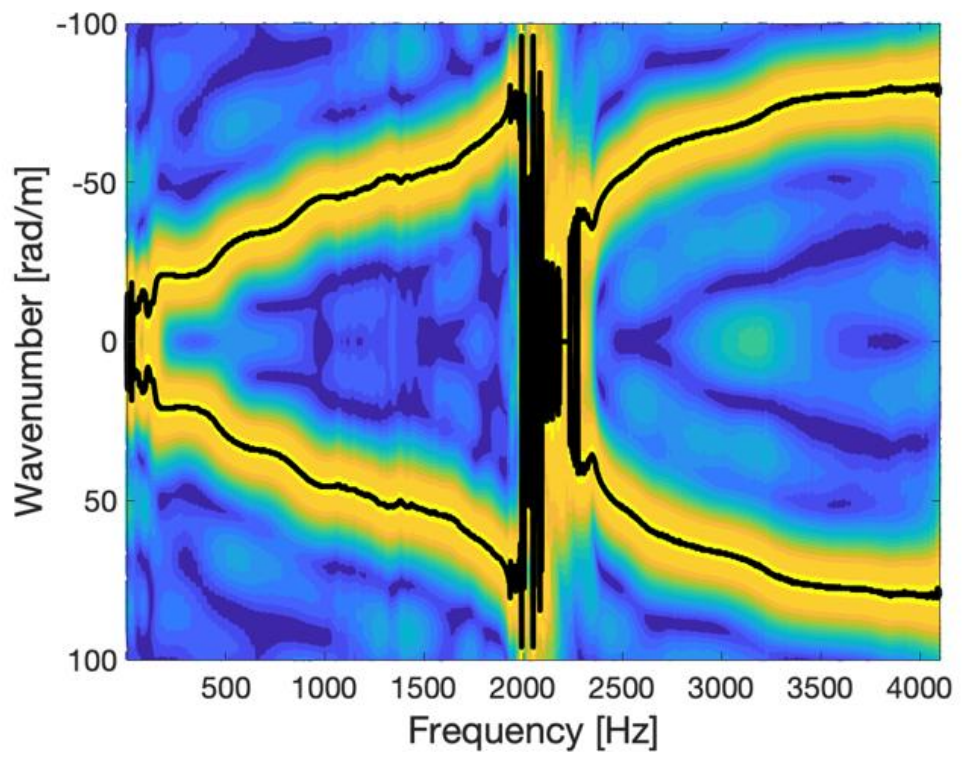

Figure 7-Typical result of the wavenumber estimation via a correlation technique. Light colours indicate higher correlation value and the black line gives the maximum correlation at each frequency. 
Once the 20 dispersion curves are estimated, the same Bayesian inference approach proposed in the previous section is applied to the experimental data in order to infer the dispersion curve for the beam with resonators. Figure 8 presents the estimated PDF of the dispersion curve from the ensemble of 20 dispersion curves obtained via the correlation technique for this one beam and it is the basis for the identification of the properties of the beam and resonators. The light yellow colour shows the wavenumbers with higher probability of representing the actual beam wavenumber, while the dark blue colour indicates the corresponding wavenumbers with lower probability. Note that the frequency band around the band gap presents higher uncertainty in the estimation of the wavenumber when compared to the other frequencies, dominated by the host structure bending. This can be attributed to the lower signal to noise ratio of the FRF at these frequencies; due to the vibration attenuation caused by the band gap, but it can also be interpreted as a statement about the break of the periodicity assumed in the nominal design, i.e. the disorder degree of the near-periodic structure that was actually manufactured.

Using the equivalent EB beam assumption, it is possible to use the wavenumber expression at lower frequencies to identify the Young's modulus of the beam. It is important to note that the correlation technique is not particularly suitable for estimating wavenumbers at very low frequencies, and these values are therefore ignored. For this particular case, the dispersion curve used to identify the Young's modulus was limited to the frequency range between $140 \mathrm{~Hz}$ (above the low frequency fluctuation of the correlation technique) and $800 \mathrm{~Hz}$ (from the transfer functions, this value is below half the natural frequency of the resonators). Figure 9 presents the identified Young's modulus and the obtained histogram for estimation at each frequency as well as the fitted Gamma distribution. This particular PDF is chosen to fit the Young's modulus because it is typically used to model non-zero positive random variables.

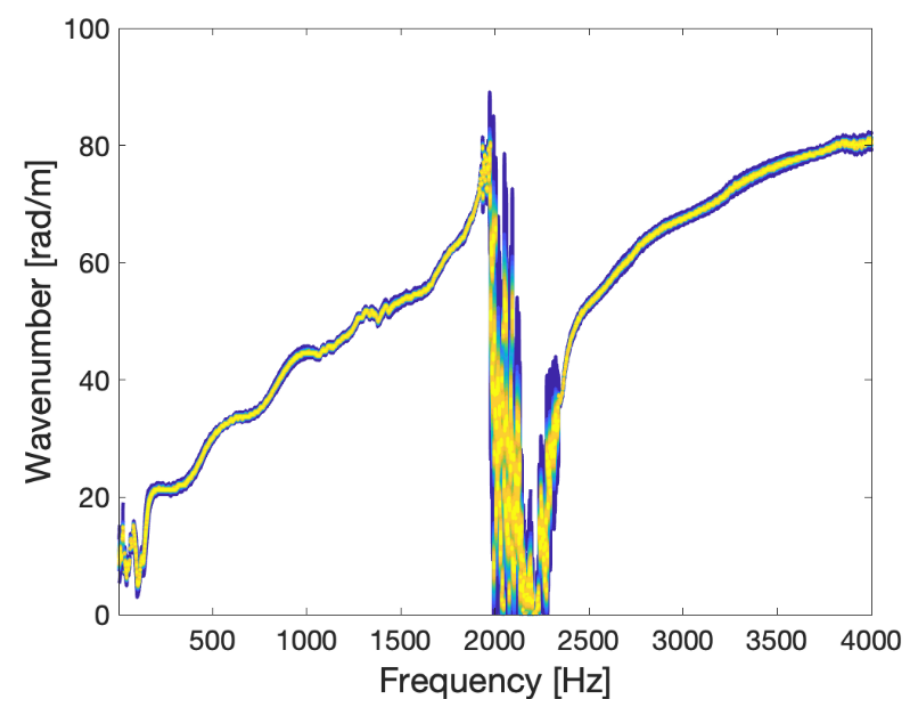

Figure 8- The updated posterior distribution from the Bayesian approach with the experimental data from the ensemble of 20 dispersion curves. Light colours indicate higher probability while dark blue indicates lower probability. 
(a)

(b)
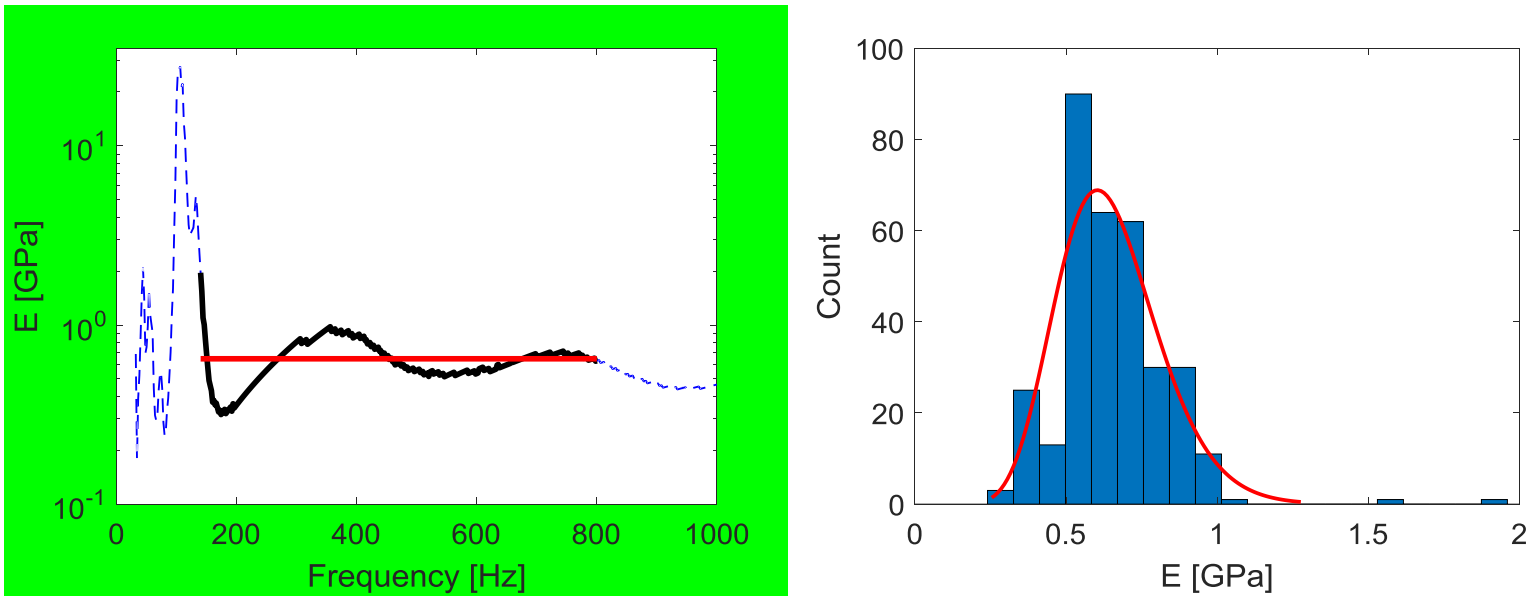

Figure 9-(a) The Young's modulus from the inferred dispersion curve as a function of the frequency (blue dashed line), the considered frequency range between $140 \mathrm{~Hz}$ and $800 \mathrm{~Hz}$ (black full line) and the average value (red full line). (b) The Young's modulus histogram and the fitted Gamma distribution (shape factor $\kappa=14.568$ and scale factor $\vartheta=4.4468 \times 10^{7}$ )

Similar to the synthetic data case, the natural frequency of the resonators $\omega_{r}$ and mass ratio $\epsilon$ are identified using a non-linear least squares approach to fit the inferred dispersion curve to the analytical model of Eq.(5), assuming the now identified Young's modulus. The fit was made in the frequency range between $1 \mathrm{kHz}$ and $2.8 \mathrm{kHz}$. These values were chosen after inspection of the dispersion curves. The lower limit was chosen in order to be roughly above $\omega_{r} / 2$, which is also the upper limit for the estimation of the Young's modulus. The upper limit is set to be below the frequency where the EB assumption breaks down and above the band gap range. A loss factor of 3\% was used in the model for the fit. The estimated values are summarized in Table 3. Typically, the 3D printing process introduces local variability on the material proprieties along the beam whilst being very accurate in reproducing the nominal geometrical properties. A procedure proposed by Beli et al. [10] was used in the manufacturing of the test sample such that the local material properties of each unit cell of the metamaterial beam can be approximated by the material properties of cube specimens printed alongside each unity cell. The elastic properties of the cube specimens are obtained by a standard non-destructive ultrasound test (see Appendix A.3. and [10]). In this work, the manufacturing variability is assumed to be uniform along the metamaterial beam, thus the average value of the parameters from the cube specimens is used as a benchmark value. The results obtained are summarized in Table 3 and show differences below $4 \%$ for the mass ratio and $1.1 \%$ for the Young's modulus.

Table 3 -Young's modulus, resonators natural frequency and mass ratio estimated from the proposed Bayesian approach and from Beli et al.[10].

\begin{tabular}{c|ccc} 
& $E[\mathrm{GPa}]$ & $f_{r}[\mathrm{~Hz}]$ & $\epsilon$ \\
\hline Bayesian approach & 0.648 & 1971.5 & 0.3135 \\
Beli et al. [10] & 0.641 & - & 0.3258 \\
error & $1.1 \%$ & - & $3.8 \%$
\end{tabular}

Figure 10 shows the experimental effective dispersion curve obtained from the proposed Bayesian inference approach and the dispersion curve calculated using the periodic analytical model 
applying the identified parameters. A very good agreement can be seen for frequencies below and at the band gap region. However, for frequencies higher than $2.8 \mathrm{kHz}$, a mismatch can be observed. This is expected since the EB assumptions are no longer valid and a Timoshenko beam model should be considered instead. Note that, although the curve fit for the parameter identification is limited to the frequency range where the EB assumptions are valid, the parameters are correctly identified.

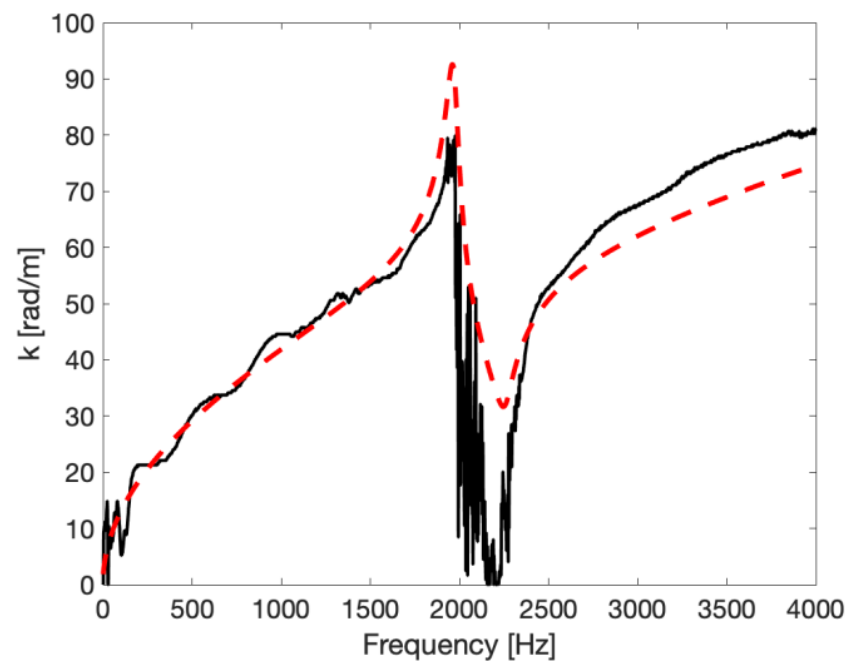

Figure 10 - Inferred experimental effective dispersion curve from the Bayes approach (solid black line) versus dispersion curve using the identified parameters on the periodic analytic model (dashed redline).

The results obtained show that the proposed Bayesian framework is capable of identifying the effective wavenumber and most probable values of some of the parameters typically of interest for vibration attenuation design using metamaterials. In order to identify local properties, i.e. the spatial profile of the mechanical properties of the metamaterial beam, a more dense set of measurement points would be necessary [33], which raises several experimental issues. For shorter distances between measurements points, shorter wavelengths are necessary to get a good signal to noise ratio between two adjacent points. This requires measurement at frequencies that are higher than the band gap, thus limiting its applicability. Kalkowski et al. [31] proposed the use of a WKB-based analytical model for estimating the local wavenumber of tapered beams using five equally spaced measurement points. Unlike the classical approach with three measurement points[30], the two extra measurements are needed because negative-going and positive-going wavenumbers are no longer the same for nonhomogeneous structures. Notwithstanding, they also point out several experimental limitations which apply in this case. Further investigation is necessary for estimating locally defined wavenumbers, also considering additional challenges such as the presence of critical sections in non-periodic structures $[10,58]$.

\section{Concluding remarks}

In this work, an investigation of the effects of the variability introduced by $3 \mathrm{D}$ printer manufacturing on the wavenumber identification of locally resonant metamaterials is presented. A combination of a correlation technique for frequency response measurements and a Bayesian framework is proposed to identify the effective wavenumber and most probable values of some of the design parameters typically of interest for vibration attenuation using metamaterials, namely the mass ratio and the resonators natural frequency. 
An analytical model assuming an equivalent homogenous structure is used to represent the periodic design of the metamaterial. The uncertainty on the estimated effective wavenumber corresponds to the degree of disorder, i.e. the breaking of the periodicity on the manufactured metastructure. This has been previously associated with the vibration attenuation performance of such structures[10].

The maximum a posteriori estimator was used to predict the most probable value for the identified parameters. First, a set of synthetic data was produced for validating the proposed approach in which Gaussian noise was added directly to the wavenumber. The identification procedure presented a maximum error of $2.1 \%$ for the Young's modulus, $0.95 \%$ for the natural frequency and $0.28 \%$ for the mass ratio of the resonators when compared to the nominal parameters of the analytical model. Then, a set of experimentally obtained frequency response functions was used to estimate wavenumber probability distribution. In this case, the identification procedure presented a maximum error of $1.1 \%$ for the Young's modulus, and 3.8\% for the mass ratio of the resonators when compared to results of a benchmark approach, i.e. a very good agreement. These results show that the proposed Bayesian approach, which relies only on frequency response measurements, can be used as a fast and efficient way of estimating the average value of the most relevant parameters for the assessment of the metastructure vibration attenuation performance. Moreover, although a correlation technique was used in this work, any other wavenumber identification procedure could have been applied and combined with the proposed approach. The approach described can be extended to other more complex metastructure designs and other types of 3D printing technologies. Understanding the effects of this variability on the wave propagation is one step towards proposing robust designs with respect to the attenuation performance.

\section{Acknowledgements}

The authors would like to thank the NT3D group from CTI Renato Archer Brazilian Institute (Campinas-SP, Brazil) for manufacturing the metamaterial beam samples through the ProEXP program, and the Nondestructive Testing Laboratory (LabEND) from FEAGRI/UNICAMP for providing the ultrasound equipment used to measure the elastic properties of the cube specimens. The authors also grateful to the São Paulo Research Foundation (FAPESP - São Paulo, Brazil), through project numbers 2014/19054-6, 2018/18774-6 and 2018/15894-0, Brazilian National Council of Research CNPq (processes number 420304/2018-5 and 231744/2013-7) the Brazilian Federal District Research Foundation - FAPDF, process number 0193.001507/2017, and the Coordination for the Improvement of Higher Education Personnel (CAPES - Brazil) for the financial support.

\section{A. Appendix}

\section{A.1. Dispersion equation with attached resonators}

In this section, the dispersion equation of a one-dimensional elastic metastructure is derived, following the approach proposed by Fabro et al. [59]. The equation of motion for a one dimensional continuous structure with $S$ periodically attached resonators can be derived as[14,36]

$$
L(x) w(x, t)+\mu \ddot{w}(x, t)-\sum_{p=1}^{S} k_{p} u_{p}(t) \delta\left(x-x_{p}\right)=p(x, t),
$$

where $L(x)$ is a linear homogeneous self-adjoint stiffness differential operator of order $2 q$, where $q>q \geq 1$ is an integer defining the order of the system, $\mu$ is the mass density per unity length $p(x, t)$ 
is the loading per unity length, $w(x, t)$ is the displacement, $u_{p}(t)$ is the displacement of each resonator attached at $x_{p}$ and $\delta(x)$ is the Dirac delta function. For each resonator, one additional equation of motion is given as

$$
m_{p} \ddot{u}_{p}(t)+k_{p} u_{p}(t)+m_{p} \ddot{w}\left(x_{p}, t\right)=0,
$$

with mass $m_{p}$ and stiffness $k_{p}$. Assuming time harmonic motion, i.e. $w(x, t)=W(x) e^{i \omega t}, p(x, t)=$ $P(x) e^{i \omega t}$, and $u_{p}(t)=U_{p} e^{i \omega t}$ for the $p^{t h}$ resonator, then

$$
\left\{\begin{array}{c}
L(x) W(x)-\omega^{2} \mu W(x)-\sum_{p=1}^{S} k_{p} U_{p} \delta\left(x-x_{p}\right)=P(x) \\
-\omega^{2} m_{p} U_{p}+k_{p} U_{p}-\omega^{2} m_{p} W\left(x_{p}\right)=0
\end{array}\right.
$$

from which

$$
U_{p}=\frac{\omega^{2}}{\omega_{p}^{2}-\omega^{2}} W\left(x_{p}\right)
$$

where $\omega_{p}^{2}=k_{p} / m_{p}$. Substituting back in Eq. A.3, then

$$
L(x) W(x)-\omega^{2} \mu W(x)-\sum_{p=1}^{S} k_{p} \frac{\omega^{2}}{\omega_{p}^{2}-\omega^{2}} W\left(x_{p}\right) \delta\left(x-x_{p}\right)=P(x),
$$

and assuming $m_{p}=\epsilon \mu \Delta l$, i.e. constant mass ratio, then

$$
L(x) W(x)-\omega^{2} \mu W(x)-\epsilon \mu \omega^{2} \sum_{p=1}^{S} \frac{\omega_{p}^{2}}{\omega_{p}^{2}-\omega^{2}} W\left(x_{p}\right) \delta\left(x-x_{p}\right) \Delta l=P(x) .
$$

Assuming identical resonators, i.e., $\omega_{p}=\omega_{r}, p=1,2, \ldots, S$, leads to

$$
L(x) W(x)-\omega^{2} \mu W(x)-\epsilon \mu \omega^{2} \frac{\omega_{r}^{2}}{\omega_{r}^{2}-\omega^{2}} \sum_{p=1}^{S} W\left(x_{p}\right) \delta\left(x-x_{p}\right) \Delta l=P(x)
$$

Assuming a large enough number of resonators, i.e.

$$
\lim _{S \rightarrow \infty} \sum_{p=1}^{S} W\left(x_{p}\right) \delta\left(x-x_{p}\right) \Delta l \approx \int_{-\infty}^{\infty} W(\xi) \delta(\xi-x) d \xi=W(x),
$$

the underlying periodic system is then effectively assumed to be an equivalent homogeneous system, which yields

$$
L(x) W(x)-\left(\omega^{2} \mu+\epsilon \mu \omega^{2} \frac{\omega_{r}^{2}}{\omega_{r}^{2}-\omega^{2}}\right) W(x)=P(x) .
$$

From this assumption, it is possible write the displacement field in terms of space harmonics, i.e. $W(x)=\widehat{W} e^{-i k x}$, thus the following dispersion equation can be found

$$
L(-i k)-\mu \omega^{2}\left(1+\epsilon \frac{1}{1-\Omega_{r}^{2}}\right)=0,
$$

where $\Omega_{\mathrm{r}}=\omega / \omega_{r}$, from which the wavenumbers can be calculated. This result is equivalent to a continuous neutralizer attached to a beam[60]. In the case of a beam undergoing flexural vibration, 
then $L(x)=d^{2} / d x^{2}\left[E I(x) d^{2} / d x^{2}\right]$, which leads to an effective wavenumber for flexural waves given by Eq. (5).

\section{A.2. Metamaterial model manufactured using SLS 3D printing}

The metamaterial structure is a rectangular cross section beam with cantilever-in-mass resonators attached at both sides, Fig. A.1, which is similar to the model investigated by Beli et al.[10]. This metamaterial beam was constructed using SLS 3D printing with 15 unit cells and geometric properties: $\Delta=22 \mathrm{~mm}, \mathrm{~b}=6 \mathrm{~mm}, \mathrm{~h}=16 \mathrm{~mm}, \mathrm{l}_{\mathrm{a}}=14 \mathrm{~mm}, \mathrm{l}_{\mathrm{b}}=2 \mathrm{~mm}, \mathrm{l}_{\mathrm{c}}=3 \mathrm{~mm}, \mathrm{l}_{\mathrm{e}}=6 \mathrm{~mm}$. The resonator presents three natural modes at the range of frequencies up to $4 \mathrm{kHz}$, which are responsible for producing the band gaps. Three modes appear in the vertical flexural motion of the beam: I resonator in torsion with small vibration attenuation, II - the resonator bending with high vibration attenuation in the excitation direction, and III - the resonator bending in the direction perpendicular to the excitation. The complex resonator flexural behaviour can be represented by stiffness and inertia matrices to take into account both displacement and rotation motions of the cantilever-in-mass resonator. In addition, because the spring-like beams that are connected the host beam to the masses/inertias are short, Beli et al. [10] used Timoshenko beam elements to describe the dynamic behaviour. However, even with this more accurate theory when compared to the Euler-Bernoulli theory, only the low frequency (acoustic mode) and band gap behaviour were well represented when compared to the experimental results. For higher frequencies (optical mode), the numerical model and measurements presented discrepancies. Therefore, the simplified analytical model described by Eq.(1) and Eq. (2) based on the Euler-Bernoulli theory is capable of estimating one of the resonator's mode and the low frequency wave behaviour.

In addition, at each unit cell side, cube specimens were printed to allow estimating the material properties of the unit cell. We assume that the material distributions (mass density and Young's modulus) along the metamaterial unit cell and along the cube specimens are similar, i.e. they have the same trend.

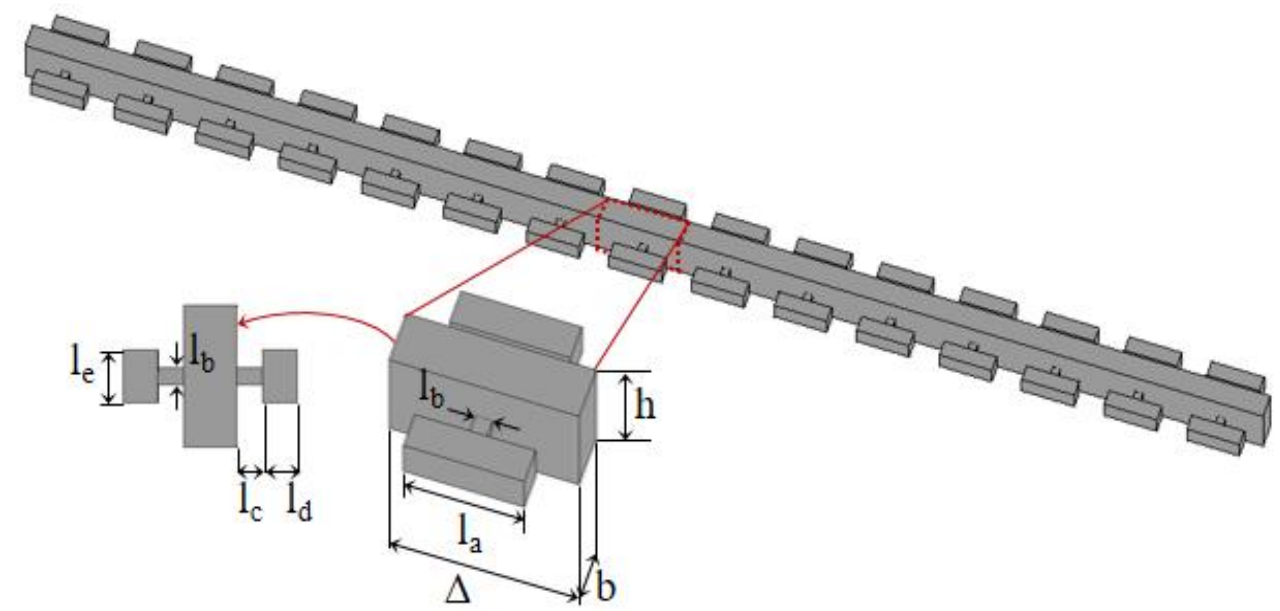

Figure A.1-Metamaterial beam and its unit cell with the geometric dimensioning.

\section{A.3. Experimental setup and frequency response measurements}

The experimental setup and measurements performed are similar to the work of Beli et al. [10]. The frequency response functions (FRF) along the beam were measured by a Laser Doppler Vibrometer (LDV) mounted on a XY-table. The input excitation was given at one end of the beam by a mini- 
hammer, and the FRFs were measured along 59 evenly spaced points (i.e., FRFS were obtained in the middle and the interfaces of each unit cell except for the excitation point) and are shown in Figure A.2 (a) and the end-to-end transfer mobility is highlighted in Figure A.2 (b) with a very distinguishable band gap between $1920 \mathrm{~Hz}$ and $2350 \mathrm{~Hz}$. The beam was supported by foams in order to achieve the free-free boundary condition. In addition, a reflective tape was applied to the beam top surface to improve the LDV measurements. These experimental FRFs were used in Section 4 for estimating the vertical flexural wavenumber of the metamaterial beam (i.e., the wave propagates along the $x$-axis with displacement field polarization in the $y$-axis).

The elastic properties of the cube specimens were obtained by a standard non-destructive ultrasound test by using $1 \mathrm{MHz}$ shear wave transducers models U8403072-U84403071 from Olympus® and the Panametrics-NDT ${ }^{\mathrm{TM}}$ EPOCH 4 system for data acquisition and signal processing, which provide the longitudinal and shear propagation times that can be related to the Young's modulus and Poisson's ratio. The mass density of the cube specimens was estimated using a precise mass balance and a digital calliper. Figure A.3 shows the estimated Young's modulus (upper) and mass density (lower) obtained with the proposed Bayesian approach (dashed red line) and from Beli et al. [10] (solid blue line).

(a)

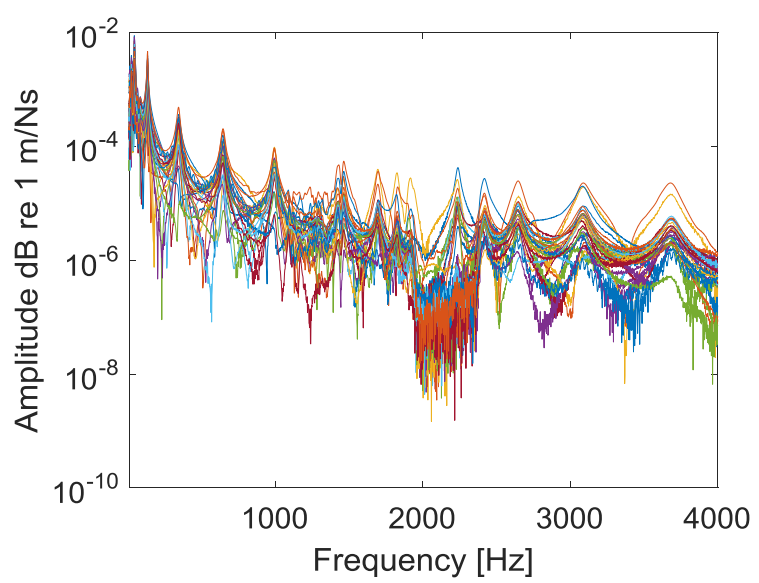

(b)

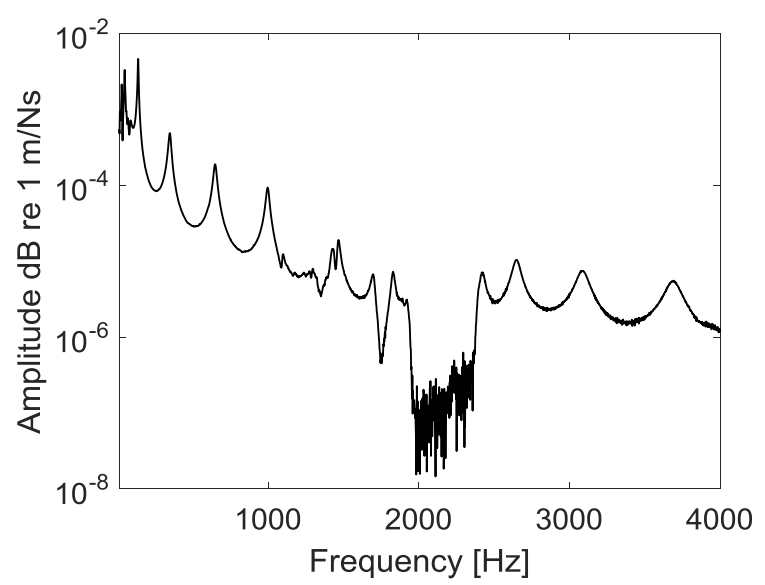

Figure A.2 - (a) Amplitude of all of the measured mobility along the metamaterial beam and (b) amplitude of the measured end-to-end transfer mobility of the metamaterial beam. 
Figure A.3 - Estimated Young's modulus (upper) and mass density (lower) from the proposed Bayesian approach (dashed red line) and from Beli et al. [10] (solid blue line).

\section{References}

[1] M.I. Hussein, M.J. Leamy, M. Ruzzene, Dynamics of Phononic Materials and Structures: Historical Origins, Recent Progress, and Future Outlook, Appl. Mech. Rev. 66 (2014) 040802040802. doi:10.1115/1.4026911.

[2] H.H. Huang, C.T. Sun, Wave attenuation mechanism in an acoustic metamaterial with negative effective mass density, New J. Phys. 11 (2009) 013003. doi:10.1088/1367-2630/11/1/013003.

[3] H. Sun, X. Du, P.F. Pai, Theory of Metamaterial Beams for Broadband Vibration Absorption, J. Intell. Mater. Syst. Struct. (2010). doi:10.1177/1045389X10375637.

[4] J. Christensen, M. Kadic, O. Kraft, M. Wegener, Vibrant times for mechanical metamaterials, MRS Commun. 5 (2015) 453-462. doi:10.1557/mrc.2015.51.

[5] S.A. Cummer, J. Christensen, A. Alù, Controlling sound with acoustic metamaterials, Nat. Rev. Mater. 1 (2016) 16001. doi:10.1038/natrevmats.2016.1.

[6] Y. Zhang, F. Zhang, Z. Yan, Q. Ma, X. Li, Y. Huang, J.A. Rogers, Printing, folding and assembly methods for forming 3D mesostructures in advanced materials, Nat. Rev. Mater. 2 (2017) 17019. doi:10.1038/natrevmats.2017.19.

[7] R. Hague, I. Campbell, P. Dickens, Implications on design of rapid manufacturing, Proc. Inst. Mech. Eng. Part C J. Mech. Eng. Sci. 217 (2003) 25-30. doi:10.1243/095440603762554587.

[8] R.D. Goodridge, C.J. Tuck, R.J.M. Hague, Laser sintering of polyamides and other polymers, Prog. Mater. Sci. 57 (2012) 229-267. doi:10.1016/j.pmatsci.2011.04.001.

[9] C.H. Hodges, J. Woodhouse, Vibration isolation from irregularity in a nearly periodic structure: Theory and measurements, J. Acoust. Soc. Am. 74 (1983) 894-905.

[10] D. Beli, A.T. Fabro, M. Ruzzene, J.R.F. Arruda, Wave attenuation and trapping in 3D printed cantilever-in-mass metamaterials with spatially correlated variability, Sci. Rep. 9 (2019) 5617. doi:10.1038/s41598-019-41999-0.

[11] J. Sanchez-Dehesa, A. Arreola-Lucas, G. Baez, F. Cervera, A. Climente, R. Mendez-Sanchez, Mechanical rainbow trapping and Bloch oscillations in chirped metallic beams, J. Acoust. Soc. Am. 141 (2017) 3810-3810. doi:10.1121/1.4988421.

[12] M. Ruzzene, A. Baz, Attenuation and localization of wave propagation in periodic rods using shape memory inserts, Smart Mater. Struct. 9 (2000) 805. doi:10.1088/0964-1726/9/6/310. 
[13] M. Rupin, F. Lemoult, G. Lerosey, P. Roux, Experimental Demonstration of Ordered and Disordered Multiresonant Metamaterials for Lamb Waves, Phys. Rev. Lett. 112 (2014) 234301. doi:10.1103/PhysRevLett.112.234301.

[14] C. Sugino, Y. Xia, S. Leadenham, M. Ruzzene, A. Erturk, A general theory for bandgap estimation in locally resonant metastructures, J. Sound Vib. 406 (2017) 104-123. doi:10.1016/j.jsv.2017.06.004.

[15] M.R. Machado, S. Adhikari, J.M.C. Dos Santos, J.R.F. Arruda, Estimation of beam material random field properties via sensitivity-based model updating using experimental frequency response functions, Mech. Syst. Signal Process. 102 (2018) 180-197. doi:10.1016/j.ymssp.2017.08.039.

[16] W.M. Bolstad, Introduction to Bayesian Statistics, 2nd Edition, 2nd edition, Wiley-Interscience, Hoboken, N.J, 2007.

[17] A. Cicirello, R.S. Langley, The vibro-acoustic analysis of built-up systems using a hybrid method with parametric and non-parametric uncertainties, J. Sound Vib. 332 (2013) 2165-2178. doi:10.1016/j.jsv.2012.05.040.

[18] S.M. Lynch, Introduction to Applied Bayesian Statistics and Estimation for Social Scientists, Springer-Verlag, New York, 2007. https://www.springer.com/gp/book/9780387712642 (accessed April 19, 2019).

[19] Y. Huang, C. Shao, B. Wu, J.L. Beck, H. Li, State-of-the-art review on Bayesian inference in structural system identification and damage assessment, Adv. Struct. Eng. 22 (2019) 1329-1351. doi:10.1177/1369433218811540.

[20] J. Ching, J.L. Beck, K.A. Porter, Bayesian state and parameter estimation of uncertain dynamical systems, Probabilistic Eng. Mech. 21 (2006) 81-96. doi:10.1016/j.probengmech.2005.08.003.

[21] W.P. Hernández, D.A. Castello, C.F.T. Matt, On the model building for transmission line cables: a Bayesian approach, Inverse Probl. Sci. Eng. 0 (2018) 1-29. doi:10.1080/17415977.2018.1436171.

[22] S. He, C.-T. Ng, Guided wave-based identification of multiple cracks in beams using a Bayesian approach, Mech. Syst. Signal Process. 84 (2017) 324-345. doi:10.1016/j.ymssp.2016.07.013.

[23] J. Yang, J. He, X. Guan, D. Wang, H. Chen, W. Zhang, Y. Liu, A probabilistic crack size quantification method using in-situ Lamb wave test and Bayesian updating, Mech. Syst. Signal Process. 78 (2016) 118-133. doi:10.1016/j.ymssp.2015.06.017.

[24] Beck J. L., Katafygiotis L. S., Updating Models and Their Uncertainties. I: Bayesian Statistical Framework, J. Eng. Mech. 124 (1998) 455-461. doi:10.1061/(ASCE)07339399(1998)124:4(455).

[25] T.G. Ritto, L.C.S. Nunes, Bayesian model selection of hyperelastic models for simple and pure shear at large deformations, Comput. Struct. $156 \quad$ (2015) 101-109. doi:10.1016/j.compstruc.2015.04.008.

[26] F. Simonetti, M.Y. Alqaradawi, Guided ultrasonic wave tomography of a pipe bend exposed to environmental conditions: A long-term monitoring experiment, NDT E Int. 105 (2019) 1-10. doi:10.1016/j.ndteint.2019.04.010.

[27] P. Huthwaite, F. Simonetti, High-resolution guided wave tomography, Wave Motion. 50 (2013) 979-993. doi:10.1016/j.wavemoti.2013.04.004.

[28] C. Claeys, N.G. Rocha de Melo Filho, L. Van Belle, E. Deckers, W. Desmet, Design and validation of metamaterials for multiple structural stop bands in waveguides, Extreme Mech. Lett. 12 (2017) 7-22. doi:10.1016/j.eml.2016.08.005.

[29] B. Van Damme, A. Zemp, Measuring Dispersion Curves for Bending Waves in Beams: A Comparison of Spatial Fourier Transform and Inhomogeneous Wave Correlation, Acta Acust. United Acust. 104 (2018) 228-234(7). doi:https://doi.org/10.3813/AAA.919164.

[30] J.M. Muggleton, M.J. Brennan, P.W. Linford, Axisymmetric wave propagation in fluid-filled pipes: wavenumber measurements in in vacuo and buried pipes, J. Sound Vib. 270 (2004) 171190. doi:10.1016/S0022-460X(03)00489-9.

[31] M.K. Kalkowski, J.M. Muggleton, E. Rustighi, An experimental approach for the determination of axial and flexural wavenumbers in circular exponentially tapered bars, J. Sound Vib. 390 (2017) 67-85. doi:10.1016/j.jsv.2016.10.018. 
[32] A. Gallina, L. Ambrozinski, P. Packo, L. Pieczonka, T. Uhl, W.J. Staszewski, Bayesian parameter identification of orthotropic composite materials using Lamb waves dispersion curves measurement:, J. Vib. Control. (2015). doi:10.1177/1077546315619264.

[33] M.R. Souza, N.S. Ferguson, Identification of bending stiffness via wavenumber estimation and Bayes inference, in: Proc. Jt. ICVRAM ISUMA UNCERTAINTIES Conf., Florianopolis, Brazil, 2018: p. 11. http://icvramisuma2018.org/cd/web/PDF/ICVRAMISUMA2018-0054.PDF (accessed May 3, 2019).

[34] N.S. Ferguson, C.R. Halkyard, B.R. Mace, K.H. Heron, The estimation of wavenumbers in twodimensional structures, in: Leuven, Belgium, 2002. https://eprints.soton.ac.uk/10023/ (accessed April 20, 2017).

[35] J. Berthaut, M.N. Ichchou, L. Jezequel, K-space identification of apparent structural behaviour, J. Sound Vib. 280 (2005) 1125-1131. doi:10.1016/j.jsv.2004.02.044.

[36] C. Sugino, S. Leadenham, M. Ruzzene, A. Erturk, On the mechanism of bandgap formation in locally resonant finite elastic metamaterials, J. Appl. Phys. 120 (2016) 134501. doi:10.1063/1.4963648.

[37] P.F. Pai, Metamaterial-based Broadband Elastic Wave Absorber, J. Intell. Mater. Syst. Struct. 21 (2010) 517-528. doi:10.1177/1045389X09359436.

[38] Z. Liu, X. Zhang, Y. Mao, Y.Y. Zhu, Z. Yang, C.T. Chan, P. Sheng, Locally Resonant Sonic Materials, Science. 289 (2000) 1734-1736. doi:10.1126/science.289.5485.1734.

[39] M.M. Sigalas, E.N. Economou, Elastic and acoustic wave band structure, J. Sound Vib. 158 (1992) 377-382. doi:10.1016/0022-460X(92)90059-7.

[40] Z. Zhong, Z. Lizhong, T. Zhihua, L. Laifeng, Z. Peiqiang, Flexural vibration of space aluminium alloy beam and plate at low temperatures, Cryogenics. 34 (1994) 489-492. doi:10.1016/S0011-2275(05)80113-4.

[41] K.-N. Chen, W.-H. Gau, Y.-C. Hu, Identification of material and geometrical parameters for microstructures by dynamic finite element model updating, Microsyst. Technol. 12 (2006) 736745. doi:10.1007/s00542-006-0110-6.

[42] N. Barbieri, R. Barbieri, L.C. Winikes, L.F. Oresten, Estimation of parameters of a three-layered sandwich beam, J. Mech. Mater. Struct. 3 (2008) 527-544. doi:10.2140/jomms.2008.3.527.

[43] S. Debruyne, D. Vandepitte, D. Moens, Identification of design parameter variability of honeycomb sandwich beams from a study of limited available experimental dynamic structural response data, Comput. Struct. 146 (2015) 197-213. doi:10.1016/j.compstruc.2013.09.004.

[44] C.K. Choi, H.H. Yoo, Stochastic inverse method to identify parameter random fields in a structure, Struct. Multidiscip. Optim. 54 (2016) 1557-1571. doi:10.1007/s00158-016-1534-y.

[45] M. Slonski, Bayesian identification of elastic parameters in composite laminates applying lamb waves monitoring, in: Proceesding 5th ECCOMAS Themat. Conf. OnComputational Methods Struct. Dyn. Earthq. Eng., M. Papadrakakis, V. Papadopoulos, V. Plevris, Crete Island, Greece, 2015: pp. 4246-4253.

[46] Z. Li, P. Lee, M. Davidson, S.E. Dosso, R. Murch, Nonlinear Bayesian inversion for estimating water pipeline dimensional and material parameters using acoustic wave dispersion, J. Sound Vib. 453 (2019) 294-313. doi:10.1016/j.jsv.2019.04.020.

[47] J.G. McDaniel, W.S. Shepard, Estimation of structural wave numbers from spatially sparse response measurements, J. Acoust. Soc. Am. 108 (2000) 1674-1682.

[48] T.G. Ritto, R. Sampaio, R.R. Aguiar, Uncertain boundary condition Bayesian identification from experimental data: A case study on a cantilever beam, Mech. Syst. Signal Process. 68-69 (2016) 176-188. doi:10.1016/j.ymssp.2015.08.010.

[49] E.T. Jaynes, Information Theory and Statistical Mechanics, Phys. Rev. 106 (1957) 620-630. doi:10.1103/PhysRev.106.620.

[50] E.T. Jaynes, Information Theory and Statistical Mechanics. II, Phys. Rev. 108 (1957) 171-190. doi:10.1103/PhysRev.108.171.

[51] J.N. Kapur, H. K. Kesavan, Entropy Optimization Principles with Applications, Academic Press, Inc., USA, 1992.

[52] P. Liu, S.-K. Au, Bayesian parameter identification of hysteretic behavior of composite walls, Probabilistic Eng. Mech. Complete (2013) 101-109. doi:10.1016/j.probengmech.2013.08.005. 
[53] T.G. Ritto, Bayesian approach to identify the bit-rock interaction parameters of a drill-string dynamical model, J. Braz. Soc. Mech. Sci. Eng. 37 (2015) 1173-1182. doi:10.1007/s40430-0140234-z.

[54] S.N. Goodman, Toward Evidence-Based Medical Statistics. 2: The Bayes Factor, Ann. Intern. Med. 130 (1999) 1005-1013. doi:10.7326/0003-4819-130-12-199906150-00019.

[55] T.J. Sullivan, Introduction to Uncertainty Quantification, First Edition, Springer, Switzerland, 2015.

[56] C. Soize, Uncertainty Quantification - An Accelerated Course with Advanced Applications in Computational Engineering, 1st ed., Elsevier, 2017.

[57] T. Bourgana, R.F. Boukadia, S. Jonckheere, C. Claeys, G. Chevallier, M. Ouisse, E. Deckers, Inverse characterization method of viscoelastic materialsusing dispersion analysis, in: Proc. Int. Conf. Noise Vib. Eng. ISMA2018 Int. Conf. Uncertain. Struct. Dyn. USD2018, Leuven, Belgium, 2018: pp. 4691-4705.

[58] A.T. Fabro, N.S. Ferguson, B.R. Mace, Wave propagation in slowly varying waveguides using a finite element approach, J. Sound Vib. 442 (2019) 308-329. doi:10.1016/j.jsv.2018.11.004.

[59] A.T. Fabro, R. Sampaio, E.S. de Cursi, Wave attenuation in a metamaterial beam assembly with uncertainties, in: Proc. XVIII Int. Symp. Dyn. Probl. Mech. DINAME2019, Buzios, Brazil, 2019.

[60] Y. Gao, M.J. Brennan, F. Sui, Control of flexural waves on a beam using distributed vibration neutralisers, J. Sound Vib. 330 (2011) 2758-2771. doi:10.1016/j.jsv.2011.01.002. 
1

2

3

4

5

6

7

8

9

10

11

12

13

14

15

16

17

18

19

20

21

22

23

24

25

26

27

28

29

30

31

32

33

34

35

36

37

38

39

40

41

42

43

44

45

46

47

48

49

50

51

52

53

54

55

56

57

58

59

60

61

62

63

64

65

Table 1 - Mechanical and geometrical properties of the metamaterial beam used for the synthetic data.

\begin{tabular}{c|cccccccc} 
Property & $\begin{array}{c}E \\
{[\mathrm{GPa}]}\end{array}$ & $\begin{array}{c}I \\
{\left[\mathrm{~m}^{4}\right]}\end{array}$ & $\eta$ & $\begin{array}{c}\rho \\
{\left[\mathrm{kg} / \mathrm{m}^{3}\right]}\end{array}$ & $\begin{array}{c}A \\
{\left[\mathrm{~m}^{2}\right]}\end{array}$ & $\begin{array}{c}f_{r} \\
{[\mathrm{~Hz}]}\end{array}$ & $\begin{array}{c}L \\
{[\mathrm{~m}]}\end{array}$ & $\epsilon$ \\
\hline Value & 1 & $2.048 \times 10^{-9}$ & 0.01 & 1000 & $9.6 \times 10^{-5}$ & 2100 & 0.33 & 0.35
\end{tabular}


1

2

3

4

5

6

7

8

9

10

11

12

13

14

15

16

17

18

19

20

21

22

23

24

25

26

27

28

29

30

31

32

33

34

35

36

37

38

39

40

41

42

43

44

45

46

47

48

49

50

51

52

53

54

55

56

57

58

59

60

61

62

63

64

65

Table 2 - Nominal and identified Young's modulus, resonator natural frequency and mass ratio from synthetic data.

\begin{tabular}{c|ccc} 
& $\begin{array}{c}E \\
f_{r}\end{array}$ & $\epsilon$ \\
& {$[\mathrm{GPa}]$} & {$[\mathrm{Hz}]$} & \\
\hline Nominal & 1.00 & 2100 & 0.350 \\
Identified & 0.98 & 2080 & 0.349 \\
error & $2.1 \%$ & $0.95 \%$ & $0.28 \%$
\end{tabular}


Table 3 -Young's modulus, resonators natural frequency and mass ratio estimated from the proposed Bayesian approach and from Beli et al.[26].

\begin{tabular}{c|ccc} 
& $E[\mathrm{GPa}]$ & $f_{r}[\mathrm{~Hz}]$ & $\epsilon$ \\
\hline Bayesian approach & 0.648 & 1971.5 & 0.3135 \\
Beli et al. [26] & 0.641 & - & 0.3258 \\
error & $1.1 \%$ & - & $3.8 \%$
\end{tabular}


1

2

3

4

5

6

7

8

9

10

11

12

13

14

15

16

17

18

19

20

21

22

23

24

25

26

27

28

29

30

31

32

33

34

35

36

37

38

39

40

41

42

43

44

45

46

47

48

49

50

51

52

53

54

55

56

57

58

59

60

61

62

63

64

65

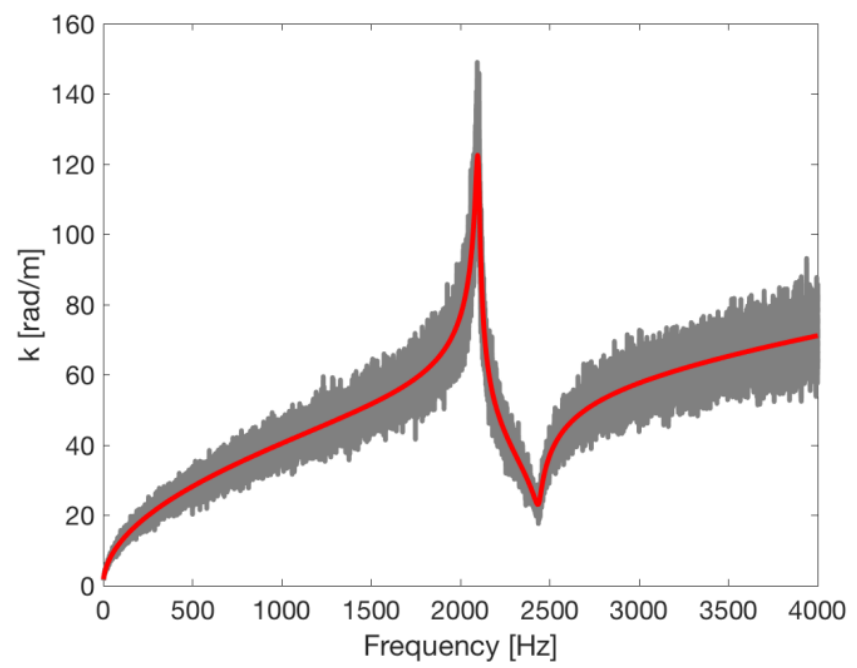

Figure 1 - Synthetic data and synthetic data polluted with noise. Nominal case $(-)$ and the twenty synthetic cases (-). 


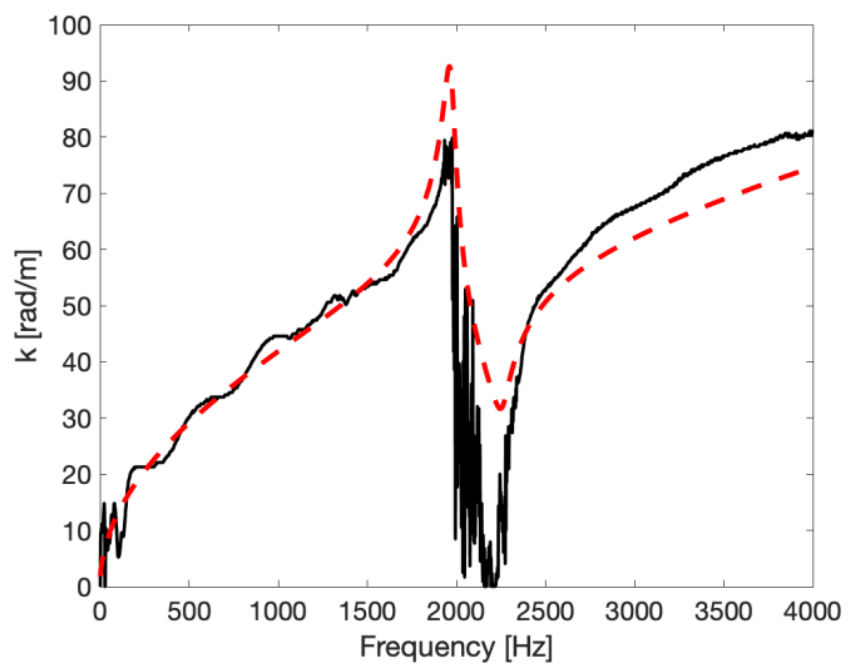

Figure 10 - Inferred experimental effective dispersion curve from the Bayes approach (solid black line) versus dispersion curve using the identified parameters on the periodic analytic model (dashed redline). 
1

2

3

4

5

6

7

8

9

10

11

12

13

14

15

16

17

18

19

20

21

22

23

24

25

26

27

28

29

30

31

32

33

34

35

36

37

38

39

40

41

42

43

44

45

46

47

48

49

50

51

52

53

54

55

56

57

58

59

60

61

62

63

64

65

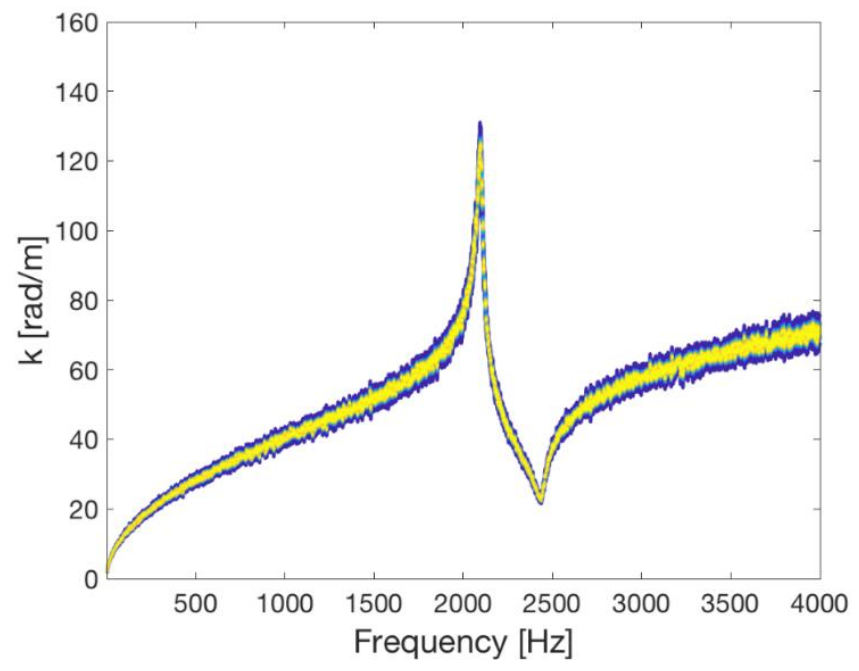

Figure 2 - Dispersion curve identified via Bayesian inference from the synthetic data. Light colours indicate higher probability while dark blue indicates lower probability. 
(a)

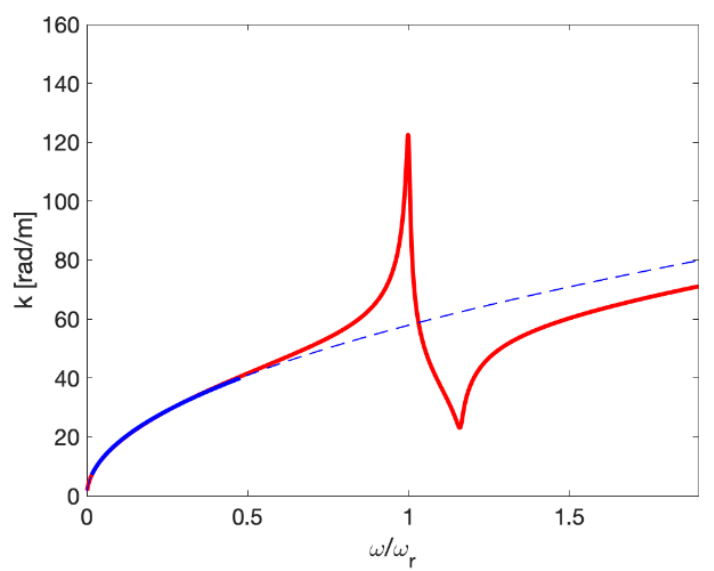

(b)

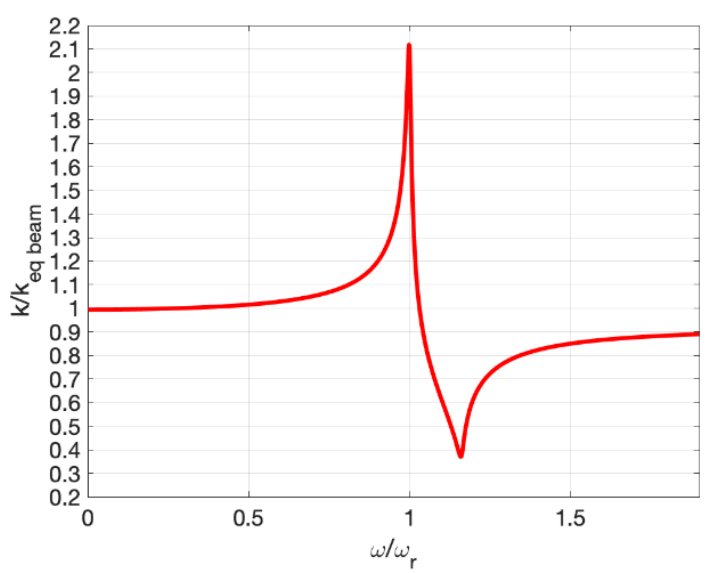

Figure 3 - (a) Dispersion curves of the beam with resonators (red full line) and for the equivalent bare beam (dashed blue line). The frequency range used to identify the Young's modulus is highlighted (solid blue line). (b) Ratio of the wavenumber of the metamaterial to that of the equivalent beam. 
(a)

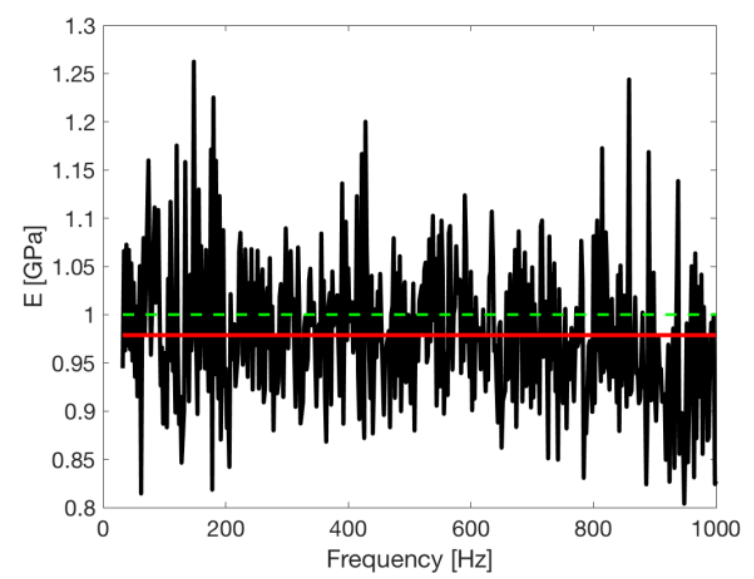

(b)

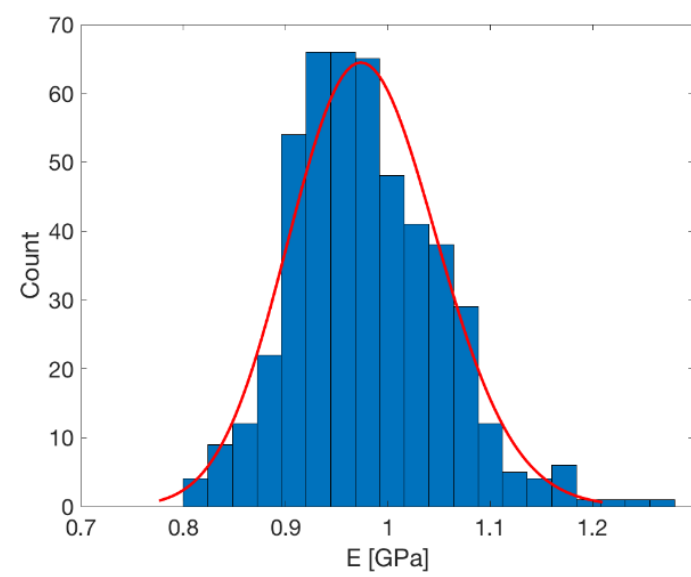

Figure 4 - (a) Young's modulus from the Bayesian inferred dispersion curve and the equivalent EB beam. Calculated (black full line), actual (dashed green line) and identified (solid red line) Young's modulus. (b) Histogram of the Young's modulus and the fitted Gamma distribution with shape factor $\kappa=183.73$ scale factor $\vartheta=5.327 \times 10^{6}$. 
1

2

3

4

5

6

7

8

9

10

11

12

13

14

15

16

17

18

19

20

21

22

23

24

25

26

27

28

29

30

31

32

33

34

35

36

37

38

39

40

41

42

43

44

45

46

47

48

49

50

51

52

53

54

55

56

57

58

59

60

61

62

63

64

65

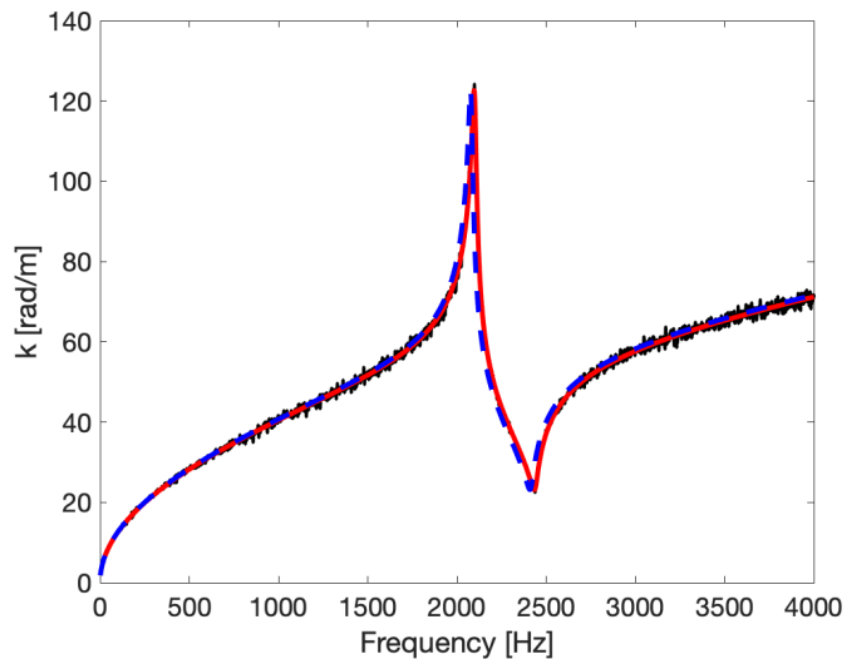

Figure 5 -Dispersion curve obtained with nominal values (dashed blue line), with the identified parameters (solid red line) and the Bayesian inferred (black line). 
1

2

3

4

5

6

7

8

10

11

12

13

14

15

16

17

18

19

20

21

22

23

24

25

26

27

28

29

30

31

32

33

34

35

36

37

38

39

40

41

42

43

44

45

46

47

48

49

50

51

52

53

54

55

56

57

58

59

60

61

62

63

64

65

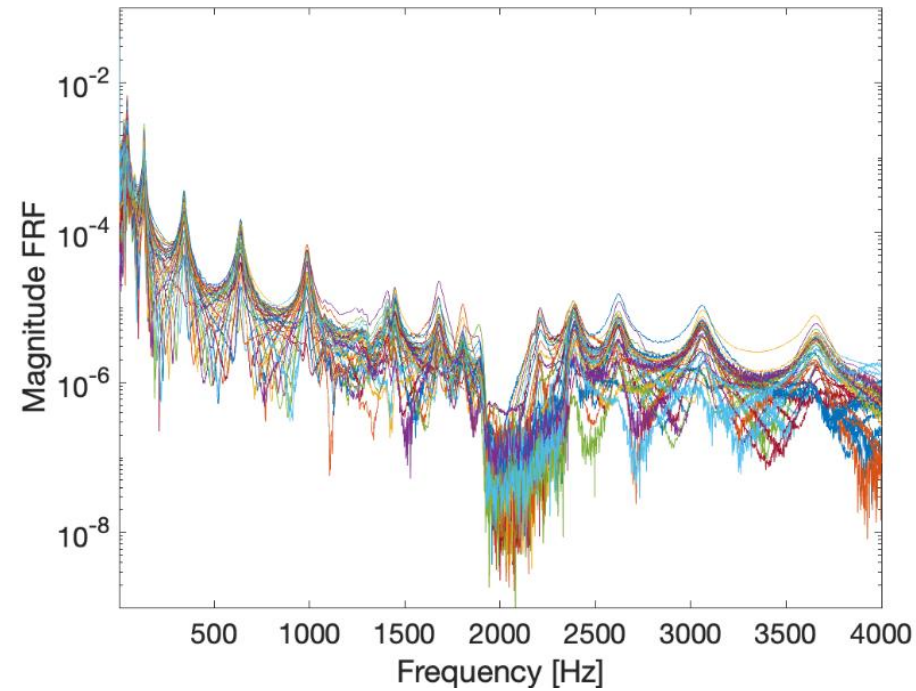

Figure 6 - Experimentally obtained FRFs. 


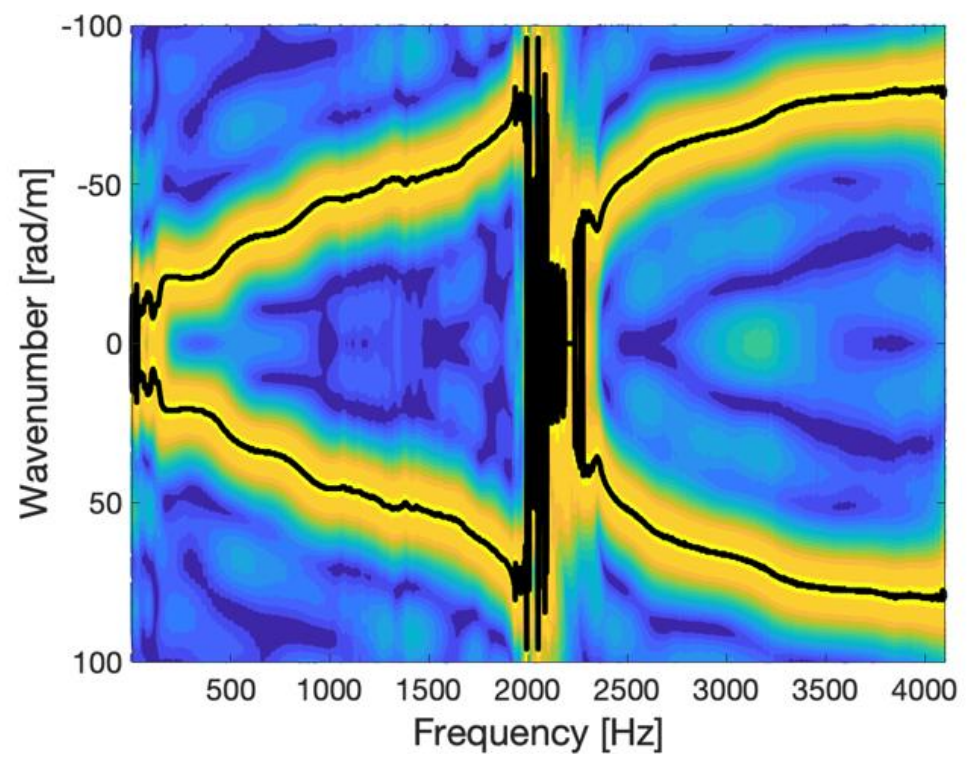

Figure 7 -Typical result of the wavenumber estimation via a correlation technique. Light colours indicate higher correlation value and the black line gives the maximum correlation at each frequency. 
1

2

3

4

5

6

7

8

9

10

11

12

13

14

15

16

17

18

19

20

21

22

23

24

25

26

27

28

29

30

31

32

33

34

35

36

37

38

39

40

41

42

43

44

45

46

47

48

49

50

51

52

53

54

55

56

57

58

59

60

61

62

63

64

65

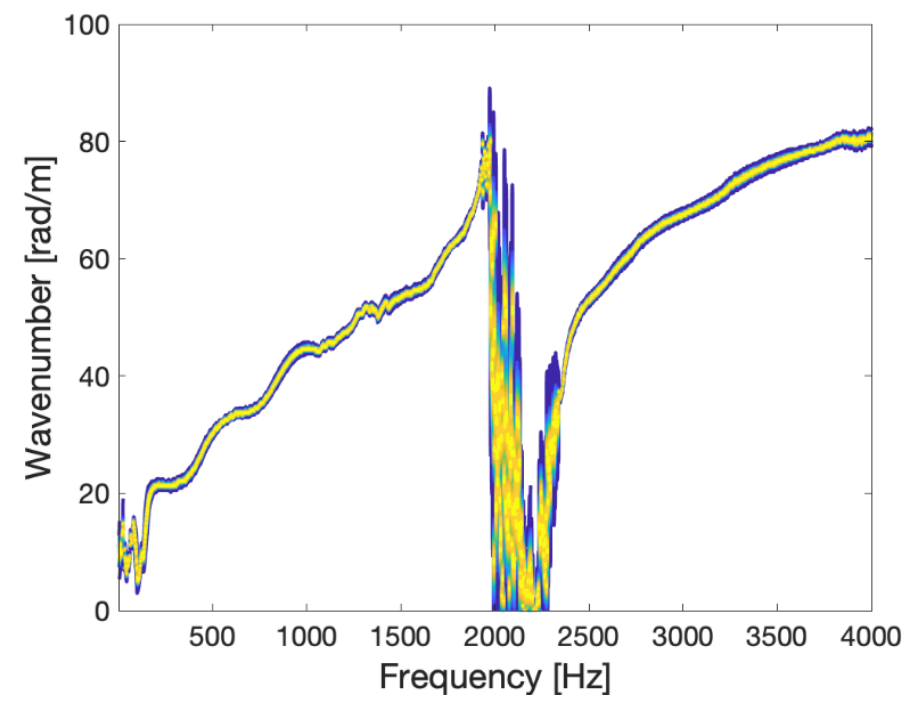

Figure 8 - The updated posterior distribution from the Bayesian approach with the experimental data from the ensemble of 20 dispersion curves. Light colours indicate higher probability while dark blue indicates lower probability. 
(a)

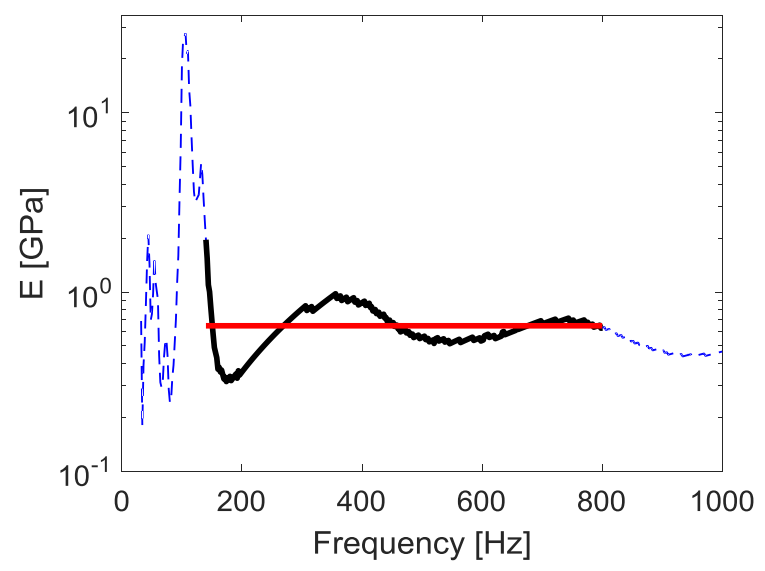

(b)

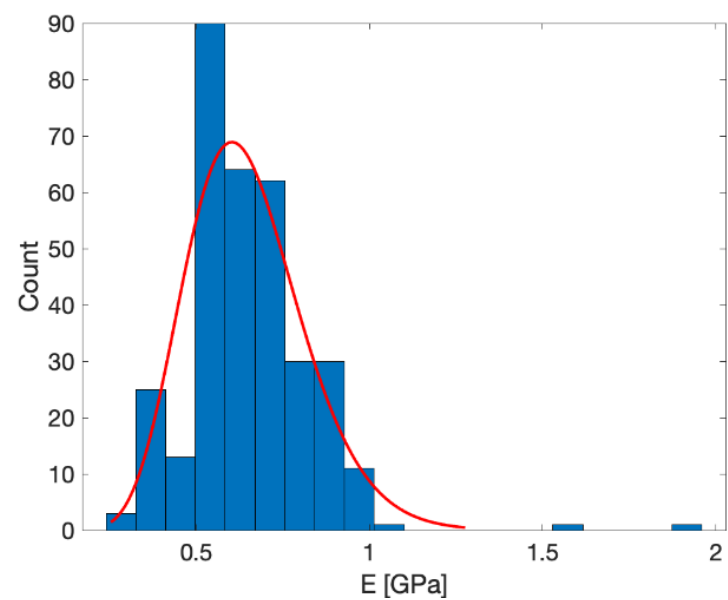

Figure 9 - (a) The Young's modulus from the inferred dispersion curve as a function of the frequency (blue dashed line), the considered frequency range between $140 \mathrm{~Hz}$ and $800 \mathrm{~Hz}$ (black full line) and the average value (red full line). (b) The Young's modulus histogram and the fitted Gamma distribution (shape factor $\kappa=14.568$ and scale factor $\vartheta=4.4468 \times 10^{7}$ ) 
1

2

3

4

5

6

7

8

10

11

12

13

14

15

16

17

18

19

20

21

22

23

24

25

26

27

28

29

30

31

32

33

34

35

36

37

38

39

40

41

42

43

44

45

46

47

48

49

50

51

52

53

54

55

56

57

58

59

60

61

62

63

64

65

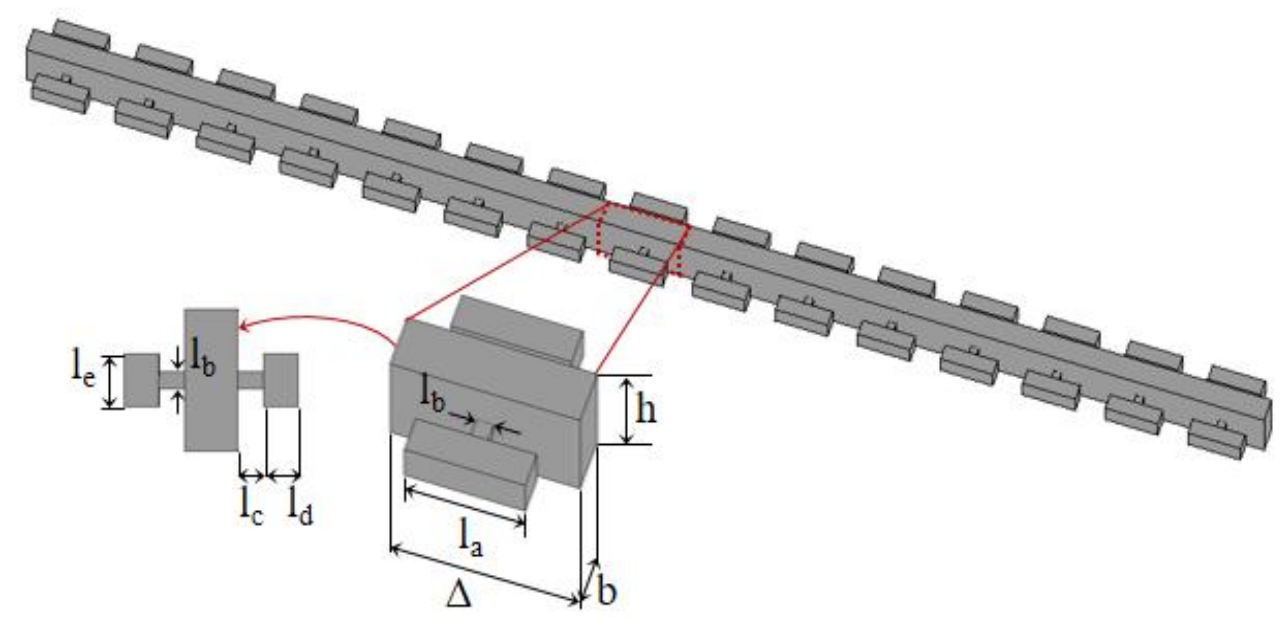

Figure A.1- Metamaterial beam and its unit cell with the geometric dimensioning. 
(a)

1

2

3

4

5

6

7

8

9

10

11

12

13

14

15

16

17

18

19

20

21

22

23

24

25

26

27

28

29

30

31

32

33

34

35

36

37

38

39

40

41

42

43

44

45

46

47

48

49

50

51

52

53

54

55

56

57

58

59

60

61

62

63

64

65

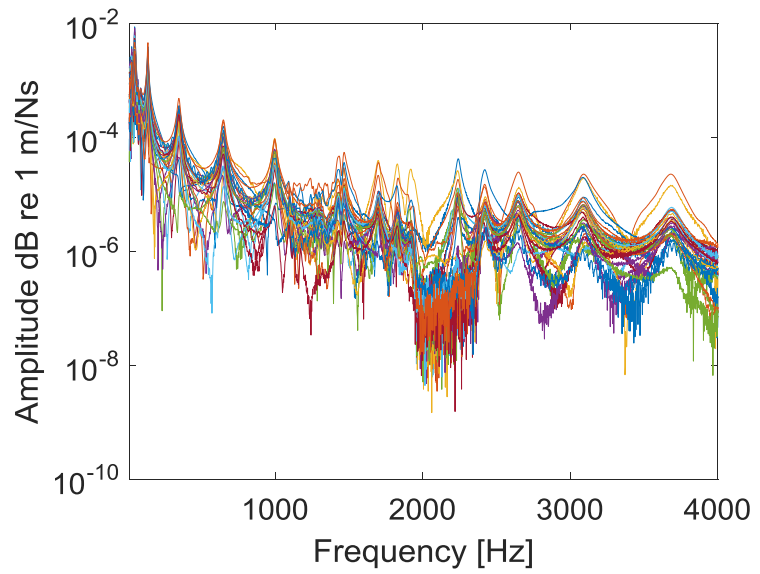

(b)

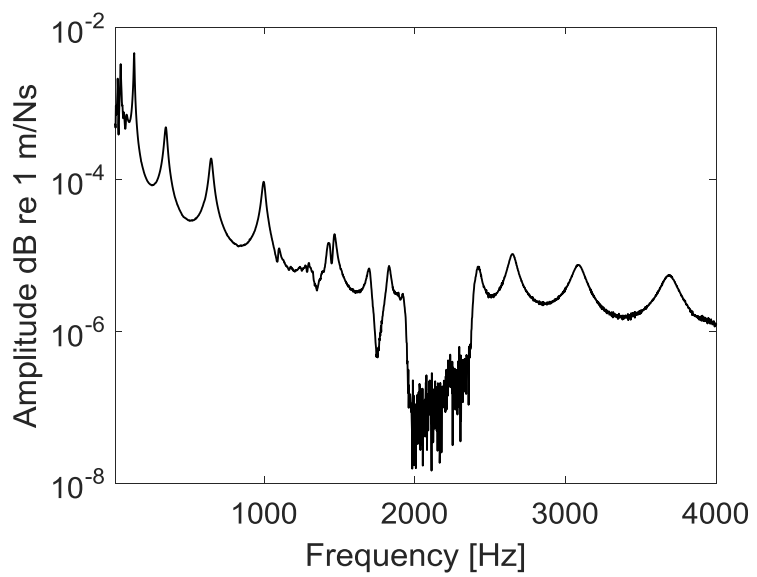

Figure A.2 - (a) Amplitude of all of the measured mobility along the metamaterial beam and (b) amplitude of the measured end-to-end transfer mobility of the metamaterial beam. 

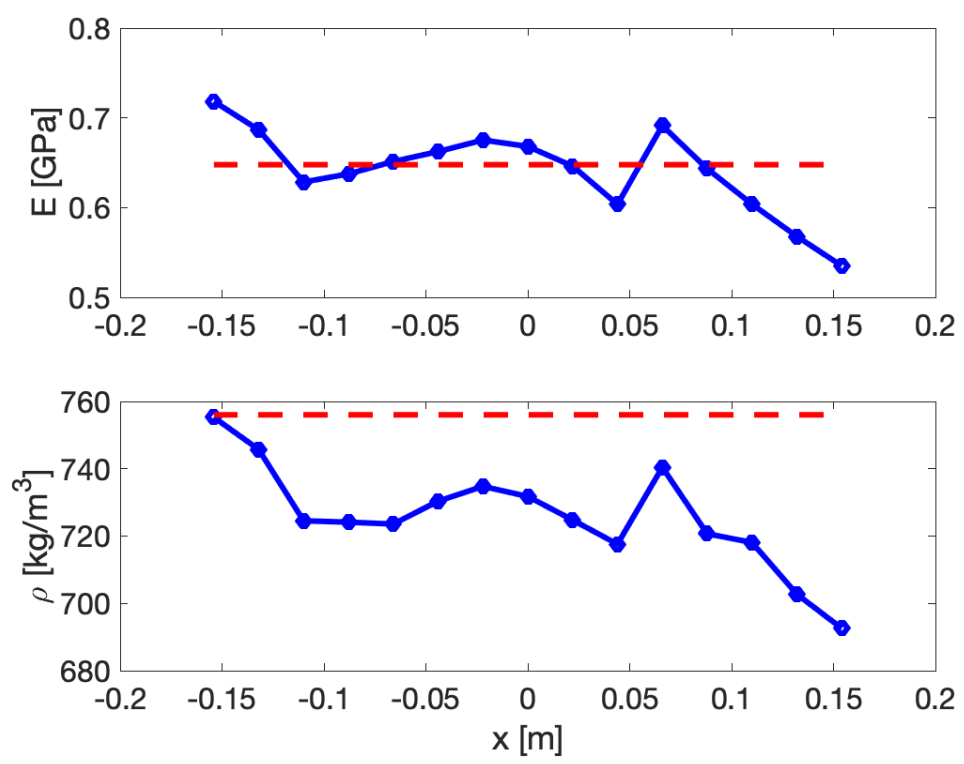

Figure A.3 - Estimated Young's modulus (upper) and mass density (lower) from the proposed Bayesian approach (dashed red line) and from Beli et al. [26] (solid blue line). 